\title{
Effect of Imperfect Channel Estimation on Transmit Diversity in CDMA Systems
}

\author{
Xiangyang Wang and Jiangzhou Wang, Senior Member, IEEE
}

\begin{abstract}
In this paper, the effect of imperfect channel estimation on the transmit diversity based on space-time block coding for the downlink of a direct-sequence code-division multiple-access system is studied. Two transmit antenna and one receiving antennas are employed. However, the results of this paper can be extended to the system with more receiving antennas. Each channel is modeled as frequency-selective Rayleigh fading and the pair of channels corresponding to two transmit antennas are mutually independent. Both spatial diversity gain and multipath diversity gain are obtained in the system. The system performance is evaluated in terms of bit-error rate under the perfect and imperfect channel estimation. A pilot-assisted channel-estimation scheme with one common spreading code sequence is exploited. It is shown that the inaccurate channel estimates suffering from multiple access and multipath interference significantly degrade the system performance and can be effectively improved by use of a simple low-pass filter. The investigation of the power ratio of pilot to data channels illustrates that the base station should dynamically adjust the transmit power of the pilot channel according to the varying system configurations in order to achieve the best performance.
\end{abstract}

Index Terms-Channel estimation, code-division multiple access (CDMA), Rayleigh fading, space-time block coding (STBC), spatial and multipath diversity, transmit diversity (TD).

\section{INTRODUCTION}

I N RECENT years, transmit diversity (TD) has been studied extensively as an effective technique to achieve spatial diversity for downlink with multiple transmit antennas at a base station (BS) rather than multiple receiving antennas at mobile terminals [1]-[4]. Since the future mobile multimedia services will be involved with high data rate, it is desirable for TD techniques to provide improvement of both link-level performance and system capacity on the high-speed downlink without increasing the total transmit power [5].

The TD scheme based on space-time block coding (STBC) proposed in [4] is quite attractive due to its simplicity. The TD is able to achieve comparable diversity gain with receiving diversity for a given number of antennas without bandwidth expansion. Due to its significance, the two-antenna TD with the STBC scheme has been adopted in the third-generation $(3 \mathrm{G})$ wireless communications standards [6], [7].

The essential virtue feature of the TD-STBC scheme [4] is its inherent coding orthogonality. This guarantees that at the receiver only simple linear decoding is required to provide the

Manuscript received June 23, 2003; revised January 31, 2004 and March 4, 2004. This project was supported by the Research Grant Council (RGC) of the Hong Kong government.

The authors are with the Department of Electrical and Electronic Engineering, The University of Hong Kong, Hong Kong (e-mail: xywang@eee.hku.hk; jwang@eee.hku.hk).

Digital Object Identifier 10.1109/TVT.2004.833635 spatial diversity gain that is equivalent to the maximum ratio receiving combining (MRRC) in the case of perfect channel estimation. However, due to multipath propagation and multiuser interference under the real radio propagation environment, the orthogonality of TD-STBC is no longer preserved, so that the pair of signals transmitted simultaneously from two antennas will cause some mutual interference terms that cannot be cancelled out by the simple linear decoding at the receiver. So far, flat fading channels have been assumed in many papers [1]-[5], [8]; however, a more realistic channel model should be the frequency-selective fading channel, especially for the $3 \mathrm{G}$ systems in which the spreading bandwidth is much greater than the coherent bandwidth of the typical cellular environment [9], [10].

In this paper, the TD-STBC under multiuser and multipath fading environment is studied for CDMA systems. A two-dimensional (2-D) RAKE-type (2-D-RAKE) receiver is utilized to collect the signal energy from multipaths, where the signals to be combined in multiple paths will first be STBC decoded. Since the TD-STBC is very sensitive to channel-estimation errors [11], [12], the impact of imperfect channel estimation on TD-STBC in the CDMA system is studied. The rest of this paper is organized as follows. Section II gives the description of basic system framework, including the transmitter, channel, and receiver models. In Section III, assuming that the ideal channel-state information (CSI) is available at the receiver, the bit-error rate (BER) of the 2-D-RAKE combining approaches are studied. Section IV investigates the effect of imperfect channel estimation on TD-STBC, where the common pilot channel is exploited to assist receivers to estimate the channel coefficients. In Section V, the system performance and optimal parameters are demonstrated by representative numerical evaluation. Finally, Section VI draws the conclusion.

\section{SySTEM MODEL}

\section{A. Transmitter Model in CDMA Downlink}

In this paper, two transmit antennas are employed at a BS while only one receiving antenna is used at each mobile station. It is assumed that there are $K$ active CDMA users in total and a common pilot signal is transmitted simultaneously from the two transmit antennas for channel estimation [6]. Thus, totally $K+1$ different spreading code sequences are required, which are mutually orthogonal within one symbol interval. Furthermore, it is assumed that all spreading codes are unit-norm complex random binary sequences with value $( \pm 1 \pm j) / \sqrt{2}$, where $j=$ 


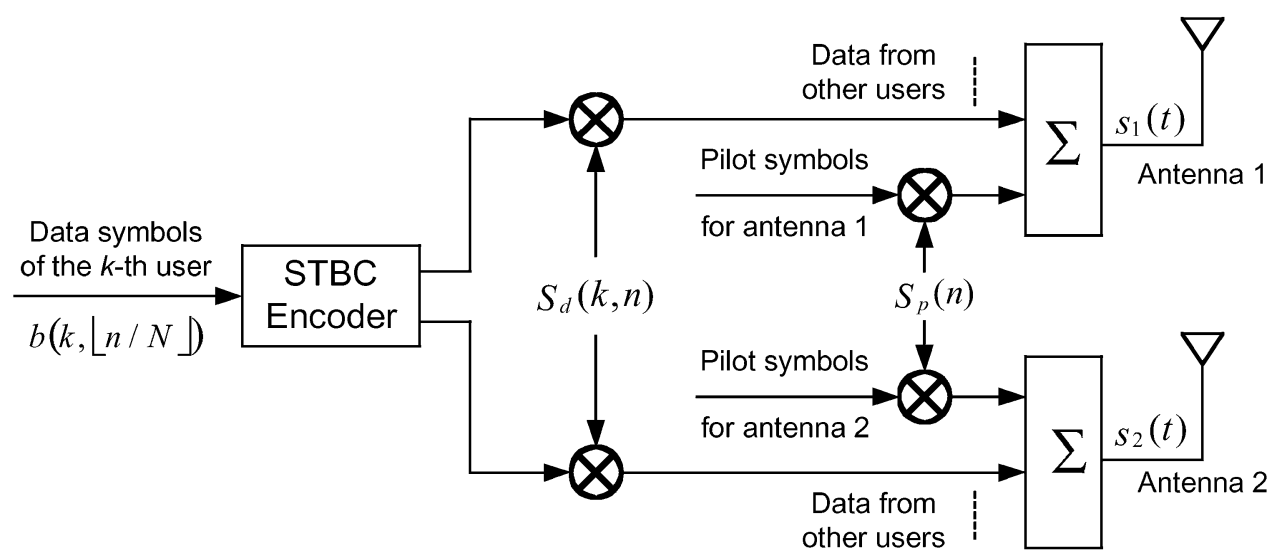

(a)

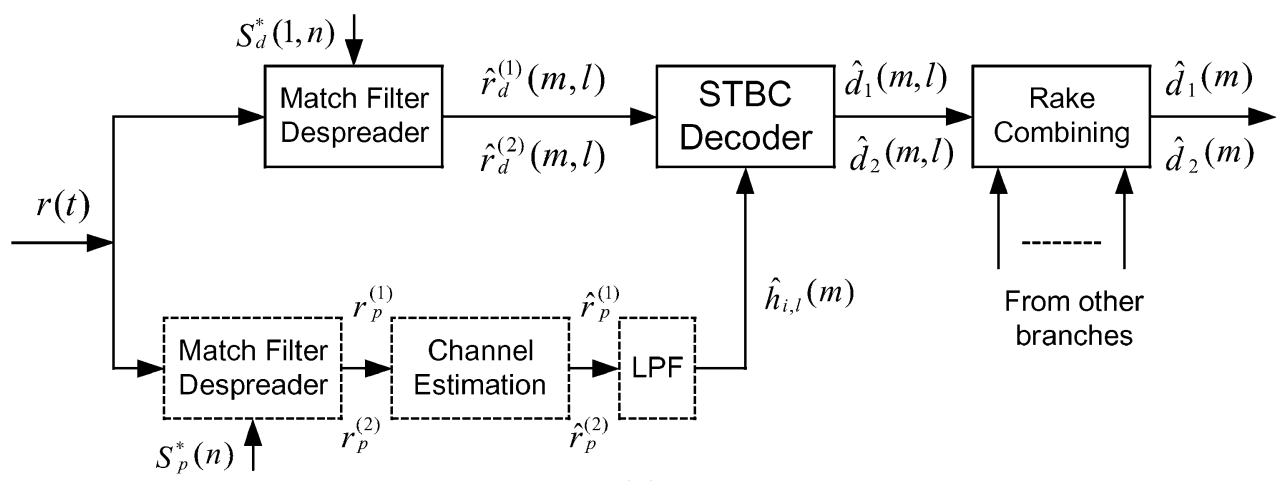

(b)

Fig. 1. Block diagrams of the transmitter and receiver.

$\sqrt{-1}$. As shown in Fig. 1, the low-pass equivalent transmitted signals at the two antennas are, respectively

$$
\begin{aligned}
s_{1}(t)= & \sum_{n=-\infty}^{\infty}\left[\sqrt{E_{c}} \cdot \sum_{k=1}^{K} b(k,\lfloor n / N\rfloor)\right. \\
& \left.\cdot S_{d}(k, n)+\sqrt{g E_{c}} \cdot S_{p}(n)\right] \cdot g\left(t-n T_{c}\right) \\
s_{2}(t)= & \sum_{n=-\infty}^{\infty}\left[\sqrt{E_{c}} \cdot \sum_{k=1}^{K} b^{\prime}(k,\lfloor n / N\rfloor)\right. \\
& \left.\cdot S_{d}(k, n)+\sqrt{g E_{c}} \cdot(-1)^{\lfloor n / N\rfloor} \cdot S_{p}(n)\right] \\
& \cdot g\left(t-n T_{c}\right)
\end{aligned}
$$

where $E_{c}$ is the chip energy of the data channel and $g$ is the power ratio of the pilot to data channels. The operation $\lfloor\cdot\rfloor$ stands for the integer part of the operand. $S_{d}(k, n)$ represents the data-channel spreading code sequence of the $k$ th user and $S_{p}(n)$ stands for the spreading code sequence of the pilot channel. $N$ is the spread factor (SF). $T_{c}$ is the chip interval and $g(t)$ is the chip waveform. For the sake of simple analysis, the binary phase-shift keying (BPSK) modulation scheme is considered and the data symbol $b(k, n)$ of the $k$ th user is assumed as real value. In (2), $b^{\prime}(k, n)$ is the STBC encoded data symbol [4], given by

$$
b^{\prime}(k,\lfloor n / N\rfloor)= \begin{cases}-b^{*}(k,\lfloor n / N\rfloor+1), & \lfloor n / N\rfloor \text { is even } \\ b^{*}(k,\lfloor n / N\rfloor-1), & \lfloor n / N\rfloor \text { is odd }\end{cases}
$$

where the superscript ${ }^{*}$ represents the complex conjugate operation.

\section{B. Channel Model}

In this paper, the discrete tap-delay-line channel model is assumed and the channel from the $i$ th transmit antenna to the receiving antenna comprises $L$ discrete resolvable paths, expressed through the channel coefficients $h_{i, l}(i=1,2$ and $l=$ $1, \ldots, L)$. The two sets of temporal multipaths corresponding to two transmit antennas experience independent but identically distributed (i.i.d.) Rayleigh fading with a constant multipath intensity profile (MIP). In other words, the second moments of the channel coefficients for all paths are the same. Therefore, it is assumed that the channel coefficients $h_{i, l}$ are complex-valued Gaussian random variables with zero mean and the same variance of $\sigma^{2}$ in both real and imaginary parts. The low-pass equivalent complex impulse response of the channel between the $i$ th transmit antenna and receiving antenna is

$$
h_{i}(t)=\sum_{l=1}^{L} h_{i, l} \cdot \delta\left(t-\tau_{l}\right)
$$

where $\tau_{l}$ is the delay of the $l$ th resolvable path, irrespective of antenna index $i$. In this paper, the path delays are treated as known constants that can be estimated by perfect acquisition and tracking algorithms. For a given temporal path index $l$, the fading coefficients $h_{1, l}$ and $h_{2, l}$ are Rayleigh distributed and independent of each other. Moreover, for a given antenna, $h_{i, l}$ and $h_{i, l^{\prime}}\left(l \neq l^{\prime}\right)$ are independent random variables. Finally, $h_{i, l}$ 
is assumed to be invariant over at least one STBC block that is refered to as every two consecutive symbols.

\section{Receiver Model}

For any mobile user, downlink signals from $K$ synchronous data channels and one pilot channel experience the same frequency-selective fading and arrive at the receiver as

$$
r(t)=\sum_{l=1}^{L}\left[h_{1, l} \cdot s_{1}\left(t-\tau_{l}\right)+h_{2, l} \cdot s_{2}\left(t-\tau_{l}\right)\right]+\eta(t)
$$

where $\eta(t)$ represents the additive white Gaussian noise (AWGN) with double-sided power spectral density (PSD) of $\eta_{0} / 2$.

The signal of each path can be resolved by a matched filter (MF) with the local delayed spreading sequence. Within the context of wide-band CDMA, it is assumed that multipath delays are approximately a few chips in duration and smaller than the symbol period, so that interdata-symbol interference can be neglected. Without loss of generality, let us focus on the $m$ th STBC block. By sampling the output of the pulse-matching filter, the received signal in the first and second symbol interval of the $m$ th STBC block can be given as, respectively

$$
\begin{aligned}
u^{(1)}( & m, n) \\
= & \sum_{k=1}^{K} \sum_{l=1}^{L} \sqrt{E_{c}} \cdot S_{d}\left(k, 2 m N+n-\left\lfloor\tau_{l} / T_{c}\right\rfloor\right) \\
& \cdot\left[b_{1}(k, m) \cdot h_{1, l}-b_{2}(k, m) \cdot h_{2, l}\right] \\
& +\sum_{l=1}^{L} \sqrt{g E_{c}} \cdot S_{p}\left(2 m N+n-\left\lfloor\tau_{l} / T_{c}\right\rfloor\right) \\
& \cdot\left(h_{1, l}+h_{2, l}\right)+\eta^{(1)}(m, n) \\
u^{(2)}( & m, n) \\
= & \sum_{k=1}^{K} \sum_{l=1}^{L} \sqrt{E_{c}} \cdot S_{d}\left[k,(2 m+1) \cdot N+n-\left\lfloor\tau_{l} / T_{c}\right\rfloor\right] \\
& \cdot\left[b_{2}(k, m) \cdot h_{1, l}+b_{1}(k, m) \cdot h_{2, l}\right] \\
& +\sum_{l=1}^{L} \sqrt{g E_{c}} \cdot S_{p}\left[(2 m+1) \cdot N+n-\left\lfloor\tau_{l} / T_{c}\right\rfloor\right] \\
& \cdot\left(h_{1, l}-h_{2, l}\right)+\eta^{(2)}(m, n)
\end{aligned}
$$

where $n=0,1, \ldots, N-1$ and $b_{j}(k, m)(j=1,2)$ stands for the $j$ th data bit of the $k$ th active user transmitted in the $m$ th STBC block. $\eta^{(j)}(m, n)(j=1,2)$ is the sampled AWGN in the $j$ th symbol period of the $m$ th STBC block.

\section{Receiver With Perfect ChanNel Estimation}

In this section, it is assumed that the ideal CSI is available at the receiver so that the pilot signal transmission is not required, i.e., $g=0$ in (1) and (2). Therefore, (6) and (7) reduce to

$$
\begin{aligned}
& u^{(1)}(m, n) \\
& =\sum_{k=1}^{K} \sum_{l=1}^{L} \sqrt{E_{c}} \cdot S_{d}\left(k, 2 m N+n-\left\lfloor\tau_{l} / T_{c}\right\rfloor\right) \\
& \quad \cdot\left[b_{1}(k, m) \cdot h_{1, l}-b_{2}(k, m) \cdot h_{2, l}\right] \\
& \quad+\eta^{(1)}(m, n)
\end{aligned}
$$

$$
\begin{aligned}
u^{(2)}(m, n) & \\
= & \sum_{k=1}^{K} \sum_{l=1}^{L} \sqrt{E_{c}} \cdot S_{d}\left[k,(2 m+1) \cdot N+n-\left\lfloor\tau_{l} / T_{c}\right\rfloor\right] \\
& \cdot\left[b_{2}(k, m) \cdot h_{1, l}+b_{1}(k, m) \cdot h_{2, l}\right]+\eta^{(2)}(m, n) .
\end{aligned}
$$

The first user $(k=1)$ is assumed to be the desired user. Assuming the perfect chip-timing synchronization and the local despreading code sequence locked to the $\hat{l}$ th resolvable path, the data channel of the first user at the $\hat{l}_{\text {th }}$ path is despread during the first and second symbol period of the $m$ th STBC block as

$$
\begin{aligned}
r_{d}^{(1)}(m, \hat{l})= & \sum_{n=0}^{N-1} u^{(1)}(m, n) \\
& \cdot S_{d}^{*}\left(1,2 m N+n-\left\lfloor\tau_{\hat{l}} / T_{c}\right\rfloor\right) \\
= & \sqrt{E_{c}} \cdot N \cdot\left[b_{1}(1, m)\right. \\
& \left.\cdot h_{1, \hat{l}}-b_{2}(1, m) \cdot h_{2, \hat{l}}\right]+\eta_{d}^{(1)}(m, \hat{l}) \\
& +\sum_{k=1}^{K} \sum_{l=1}^{L} \sqrt{E_{c}} \cdot R_{d, d}^{(1)}(m, k ; l, \hat{l}) \\
& \cdot\left[b_{1}(k, m) \cdot h_{1, l}-b_{2}(k, m) \cdot h_{2, l}\right] \\
& N-1 \\
r_{d}^{(2)}(m, \hat{l})= & \sum_{n=0}^{(2)}(m, n) \\
& \cdot S_{d}^{*}\left[1,(2 m+1) \cdot N+n-\left\lfloor\tau_{\hat{l}} / T_{c}\right\rfloor\right] \\
= & \sqrt{E_{c}} \cdot N \cdot\left[b_{2}(1, m)\right. \\
& \left.\cdot h_{1, \hat{l}}+b_{1}(1, m) \cdot h_{2, \hat{l}}\right]+\eta_{d}^{(2)}(m, \hat{l}) \\
& +\sum_{k=1}^{K} \sum_{\substack{l=1 \\
l \neq \hat{l}}}^{L} \sqrt{E_{c}} \cdot R_{d, d}^{(2)}(m, k ; l, \hat{l}) \\
& \cdot\left[b_{2}(k, m) \cdot h_{1, l}+b_{1}(k, m) \cdot h_{2, l}\right] .
\end{aligned}
$$

The background AWGN components $\eta_{d}^{(j)}(m, \hat{l})(j=1,2)$ are given by

$$
\begin{aligned}
\eta_{d}^{(j)}(m, \hat{l})= & \sum_{n=0}^{N-1} \eta^{(j)}(m, n) \\
& \cdot S_{d}^{*}\left[1,(2 m+j-1) \cdot N+n-\left\lfloor\tau_{\hat{l}} / T_{c}\right\rfloor\right] .
\end{aligned}
$$

$R_{d, d}^{(j)}(m, k ; l, \hat{l})(j=1,2)$ is the $N$-length discrete aperiodic correlation function [13] of two time-delayed data-channel spreading-code sequences used in the $j$ th symbol period of the $m$ th STBC block given by

$$
\begin{array}{r}
R_{d, d}^{(j)}(m, k ; l, \hat{l})=\sum_{n=0}^{N-1} S_{d}\left[k,(2 m+j-1) \cdot N+n-\left\lfloor\tau_{l} / T_{c}\right\rfloor\right] \\
\cdot S_{d}^{*}\left[1,(2 m+j-1) \cdot N+n-\left\lfloor\tau_{\hat{l}} / T_{c}\right\rfloor\right] .
\end{array}
$$

Since two different spreading-code sequences with the same time delay are orthogonal over one symbol interval, $R_{d, d}^{(j)}(m, k ; l, \hat{l})=0$ when $l=\hat{l}$ and $k \neq 1$. Otherwise, when $l \neq \hat{l}$, the two code sequences are nonorthogonal and $R_{d, d}^{(j)}(m, k ; l, \hat{l})$ is modeled as an i.i.d. random variable with zero mean and variance $\operatorname{Var}\left\{R_{d, d}^{(j)}(m, k ; l, \hat{l})\right\}=N$.

With respect to the $\hat{l}$ th resolvable path, it is assumed that the ideal CSI $h_{i, \hat{l}}$ is available at the receiver; thus, the random vari- 
able of the first estimated data bit in the $m$ th STBC block is constructed through STBC decoding [4] as

$$
\begin{aligned}
d_{1}(m, \hat{l})= & r_{d}^{(1)}(m, \hat{l}) \cdot h_{1, \hat{l}}^{*}+r_{d}^{(2)^{*}}(m, \hat{l}) \cdot h_{2, \hat{l}} \\
= & \sqrt{E_{c}} \cdot N \cdot b_{1}(1, m) \cdot\left(\left|h_{1, \hat{l}}\right|^{2}+\left|h_{2, \hat{l}}\right|^{2}\right) \\
& +\eta_{d}^{(1)}(m, \hat{l}) \cdot h_{1, \hat{l}}^{*}+\eta_{d}^{(2)^{*}}(m, \hat{l}) \cdot h_{2, \hat{l}} \\
& +\sum_{k=1}^{K} \sum_{\substack{l=1 \\
l \neq \hat{l}}}^{L}\left\{\sqrt{E_{c}} \cdot R_{d, d}^{(1)}(m, k ; l, \hat{l})\right. \\
& \cdot\left[b_{1}(k, m) \cdot h_{1, l}-b_{2}(k, m) \cdot h_{2, l}\right] \cdot h_{1, \hat{l}}^{*} \\
& +\sqrt{E_{c}} \cdot R_{d, d}^{(2) *}(m, k ; l, \hat{l}) \\
& \left.\cdot\left[b_{1}(k, m) \cdot h_{2, l}^{*}+b_{2}(k, m) \cdot h_{1, l}^{*}\right] \cdot h_{2, \hat{l}}\right\} \\
= & E_{1}(m, \hat{l})+N_{1}(m, \hat{l})+I_{1}(m, \hat{l})
\end{aligned}
$$

where $E_{1}(m, \hat{l})$ is the desired signal component, $N_{1}(m, \hat{l})$ is the background AWGN component, and $I_{1}(m, \hat{l})$ is the multiple access and multipath interference component, respectively, given by

$$
\begin{aligned}
E_{1}(m, \hat{l})= & \sqrt{E_{c}} \cdot N \cdot b_{1}(1, m) \cdot\left(\left|h_{1, \hat{l}}\right|^{2}+\left|h_{2, \hat{l}}\right|^{2}\right) \\
N_{1}(m, \hat{l})= & \eta_{d}^{(1)}(m, \hat{l}) \cdot h_{1, \hat{l}}^{*}+\eta_{d}^{(2)^{*}}(m, \hat{l}) \cdot h_{2, \hat{l}} \\
I_{1}(m, \hat{l})= & \sum_{k=1}^{K} \sum_{\substack{l=1 \\
l \neq \hat{l}}}^{L}\left\{\sqrt{E_{c}} \cdot R_{d, d}^{(1)}(m, k ; l, \hat{l})\right. \\
& \cdot\left[b_{1}(k, m) \cdot h_{1, l}-b_{2}(k, m) \cdot h_{2, l}\right] \cdot h_{1, \hat{l}}^{*} \\
& +\sqrt{E_{c}} \cdot R_{d, d}^{(2) *}(m, k ; l, \hat{l}) \\
& \left.\cdot\left[b_{1}(k, m) \cdot h_{2, l}^{*}+b_{2}(k, m) \cdot h_{1, l}^{*}\right] \cdot h_{2, \hat{l}}\right\} .
\end{aligned}
$$

On the other hand, the random variable of the second estimated data bit can also be constructed as [4]

$$
d_{2}(m, \hat{l})=r_{d}^{(2)}(m, \hat{l}) \cdot h_{1, \hat{l}}^{*}-r_{d}^{(1)^{*}}(m, \hat{l}) \cdot h_{2, \hat{l}}
$$

Since the data bits $b_{j}(k, m)(j=1,2)$ are i.i.d. binary random variables taking values \pm 1 and $R_{d, d}^{(j)}(m, k ; l, \hat{l})$ are i.i.d. random variables with zero-mean and variance $N$, thus conditioned on $h_{1, \hat{l}}$ and $h_{2, \hat{l}}, I_{1}(m, \hat{l})$ is a random variable with zero mean and the variance given by

$$
\begin{aligned}
\operatorname{Var}\left\{I_{1}(m, \hat{l})\right\}= & E_{c} \cdot N \cdot \sum_{k=1}^{K} \sum_{\substack{l=1 \\
l \neq \hat{\imath}}}^{L} E\left\{\left|h_{1, l}\right|^{2}+\left|h_{2, l}\right|^{2}\right\} \\
& \cdot\left(\left|h_{1, \hat{l}}\right|^{2}+\left|h_{2, \hat{l}}\right|^{2}\right) \\
= & E_{c} \cdot N \cdot K \cdot(L-1) \\
& \cdot 4 \sigma^{2} \cdot\left(\left|h_{1, \hat{\imath}}\right|^{2}+\left|h_{2, \hat{l}}\right|^{2}\right)
\end{aligned}
$$

where $\sigma^{2}=(1 / 2) E\left\{\left|h_{i, l}\right|^{2}\right\}$. The background AWGN component $N_{1}(m, \hat{l})$ is a zero-mean Gaussian variable with variance

$$
\operatorname{Var}\left\{N_{1}(m, \hat{l})\right\}=N \cdot \frac{\eta_{0}}{2} \cdot\left(\left|h_{1, \hat{l}}\right|^{2}+\left|h_{2, \hat{l}}\right|^{2}\right) .
$$

Since the data bits from $K$ active users and the channelfading coefficients along $L$ resolvable paths are independent random variables, $d_{1}(m, \hat{l})$ in (14) is the sum of many independent random variables and, hence, can be approximated as a conditional Gaussian variable. Therefore, conditioned on the channel-fading coefficients $h_{i, \hat{l}}$ and the data bit $b_{1}(1, m)$, the random variable $d_{1}(m, \hat{l})$ is a Gaussian random variable with the mean and variance

$$
\begin{aligned}
E\left\{d_{1}(m, \hat{l})\right\}= & E_{1}(m, \hat{l}) \\
\operatorname{Var}\left\{d_{1}(m, \hat{l})\right\}= & \operatorname{Var}\left\{N_{1}(m, \hat{l})\right\}+\operatorname{Var}\left\{I_{1}(m, \hat{l})\right\} \\
= & {\left[E_{c} \cdot N \cdot K \cdot(L-1) \cdot 4 \sigma^{2}+N \cdot \frac{\eta_{0}}{2}\right] } \\
& \cdot\left(\left|h_{1, \hat{l}}\right|^{2}+\left|h_{2, \hat{l}}\right|^{2}\right) .
\end{aligned}
$$

Similarly, the conditional statistics of $d_{2}(m, \hat{l})$ can be derived.

After despreading and STBC decoding at each path, a RAKE-type receiver is exploited to combine the signal energy from $L_{c}\left(L_{c} \leq L\right)$ selected paths. This can be regarded as a 2-D-RAKE combiner that collects both spatial and path diversity gain benefiting from TD-STBC and DS-CDMA merits, respectively. Note that the STBC decoding at each path conducts essentially maximal ratio combining (MRC) at the first dimension in the spatial domain.

\section{A. Maximal Ratio-Equal Gain Combiner}

In the maximal ratio-equal gain-combining (MR-EGC) receiver, the signals of first $L_{c}$ arriving paths among total $L$ resolvable paths are selected and combined. Assuming that the fading of each path is independent and that the random variables in distinct branches of the 2-D-RAKE are independent from each other, the output of the 2-D-RAKE combiner can be represented as

$$
d_{j}(m)=\sum_{l=1}^{L_{c}} d_{j}(m, l) .
$$

When $L_{c}=L$, the signals from all resolvable paths are combined. The decision variable $d_{j}(m)(j=1,2)$ is a Gaussian variable with conditional mean and variance given by

$$
\begin{aligned}
E\left\{d_{j}(m)\right\} & =\sum_{l=1}^{L_{c}} E\left\{d_{j}(m, l)\right\} \\
& =\sqrt{E_{c}} \cdot N \cdot b_{j}(1, m) \cdot \zeta \\
\operatorname{Var}\left\{d_{j}(m)\right\} & =\sum_{l=1}^{L_{c}} \operatorname{Var}\left\{d_{j}(m, l)\right\} \\
& =\left[E_{c} \cdot N \cdot K \cdot(L-1) \cdot 4 \sigma^{2}+N \cdot \frac{\eta_{0}}{2}\right] \cdot \zeta
\end{aligned}
$$

where $\zeta$ is defined as

$$
\zeta=\sum_{l=1}^{L_{c}}\left(\left|h_{1, l}\right|^{2}+\left|h_{2, l}\right|^{2}\right) .
$$


Therefore, conditioned on the instantaneous fading channel amplitudes of the multipaths, the BER can be obtained by [14]

$$
P_{e, \mathrm{EGC}}(\gamma)=Q\left(\left\{\frac{\left|E\left\{d_{j}(m)\right\}\right|^{2}}{\operatorname{Var}\left\{d_{j}(m)\right\}}\right\}^{1 / 2}\right)=Q(\sqrt{2 \gamma})
$$

where $Q(x)=(1 / \sqrt{2 \pi}) \int_{x}^{\infty} \exp \left(-t^{2} / 2\right) d t$ and by definition

$$
\gamma=\frac{E_{c} \cdot N \cdot \zeta}{E_{c} \cdot K \cdot(L-1) \cdot 8 \sigma^{2}+\eta_{0}} .
$$

Since $h_{1, l}$ and $h_{2, l}$ are independent from each other, the summed random variable $\zeta$ in (26) follows the chi-square or gamma distribution with $4 L_{c}$ degrees of freedom. The probability density function (pdf) of $\gamma$ in (28) is given by [15]

$$
p_{\gamma}(\gamma)=\frac{1}{\bar{\gamma}_{c}^{2 L_{c}} \cdot \Gamma\left(2 L_{c}\right)} \cdot \gamma^{2 L_{c}-1} \cdot \exp \left(-\frac{\gamma}{\bar{\gamma}_{c}}\right)
$$

where by definition

$$
\bar{\gamma}_{c}=\frac{E_{c} \cdot N \cdot\left(2 \sigma^{2}\right)}{E_{c} \cdot K \cdot(L-1) \cdot\left(8 \sigma^{2}\right)+\eta_{0}} .
$$

The resultant BER can be obtained by averaging the conditional BER $P_{e, \mathrm{EGC}}(\gamma)$ in (27) over the pdf of $\gamma$ in (29), [15], i.e.,

$$
\begin{aligned}
P_{e, \mathrm{EGC}} & =\int_{0}^{\infty} P_{e, \mathrm{EGC}}(\gamma) \cdot p_{\gamma}(\gamma) \cdot d \gamma \\
& =\left(\frac{1-\mu}{2}\right)^{2 L_{c}} \cdot \sum_{l=0}^{2 L_{c}-1}\left(\begin{array}{c}
2 L_{c}-1+l \\
l
\end{array}\right) \cdot\left(\frac{1+\mu}{2}\right)^{l}
\end{aligned}
$$

where by definition

$$
\mu=\sqrt{\frac{\bar{\gamma}_{c}}{1+\bar{\gamma}_{c}}}=\left[1+\frac{4 K \cdot(L-1)}{N}+\frac{1}{\bar{\gamma}_{b}}\right]^{-\frac{1}{2}}
$$

where $\bar{\gamma}_{b}$ is the average signal-to-noise ratio (SNR) per bit and is defined as

$$
\bar{\gamma}_{b}=\frac{2 E_{c} \cdot N \cdot \sigma^{2}}{\eta_{0}}
$$

In order to more clearly elaborate on the performance improvement benefiting from the spatial diversity gain due to TD-STBC and path diversity gain due to multipath RAKE combining, we define the average SNR per bit per antenna per path as

$$
\bar{\gamma}_{p}=\frac{\bar{\gamma}_{b}}{N_{T} \cdot L}=\frac{2 E_{c} \cdot N \cdot \sigma^{2}}{\eta_{0} \cdot N_{T} \cdot L}
$$

where $N_{T}$ is the number of transmit antennas and $N_{T}=2$ for TD-STBC while $N_{T}=1$ for the conventional DS-CDMA system with only one transmit antenna. This implies that the total transmit power is restricted as a constant for different system scenarios in order to evaluate and compare the performance improvement merely resulting from spatial diversity gain and multipath diversity gain.

\section{B. Maximal Ratio-Generalized Selection Combiner}

It is expected to adaptively select and combine the signals from the $L_{c}$ paths with the highest signal-to-interference-plus-noise ratio (SINR) among the total $L$ resolvable paths. Thus, a maximal ratio-generalized selection-combining (MR-GSC) 2-D-RAKE receiver that selects and combines the $L_{c}$ strongest paths is exploited next. It can be proven that $d_{1}(m, l)$ and $d_{2}(m, l)$ have the same SINR property. For the branch random variables $d_{1}(m, l)(l=1,2, \ldots, L)$, its instantaneous SINR can be derived from (21) and (22) as

$$
\frac{\left|E\left\{d_{1}(m, l)\right\}\right|^{2}}{\operatorname{Var}\left\{d_{1}(m, l)\right\}}=2 \gamma_{l}
$$

where $\gamma_{l}$ is defined by

$$
\gamma_{l}=\frac{E_{c} \cdot N}{E_{c} \cdot K \cdot(L-1) \cdot 8 \sigma^{2}+\eta_{0}} \cdot\left(\left|h_{1, l}\right|^{2}+\left|h_{2, l}\right|^{2}\right) .
$$

Since the variable $\gamma_{l}(l=1,2, \ldots, L)$ follows chi-square distribution with four degrees of freedom, its pdf and relevant cumulative distribution function (cdf) are given by

$$
\begin{aligned}
& p_{\gamma_{l}}\left(\gamma_{l}\right)=\frac{\gamma_{l}}{\bar{\gamma}_{l}^{2}} \exp \left(-\frac{\gamma_{l}}{\bar{\gamma}_{l}}\right) \\
& F_{\gamma_{l}}\left(\gamma_{l}\right)=1-\left(1+\frac{\gamma_{l}}{\bar{\gamma}_{l}}\right) \exp \left(-\frac{\gamma_{l}}{\bar{\gamma}_{l}}\right)
\end{aligned}
$$

where $\bar{\gamma}_{l}$ is defined as the same as (30), i.e., $\bar{\gamma}_{l}=\bar{\gamma}_{c}$.

For $L_{c}$ strongest paths selected from $L$ resolvable paths, $\gamma_{1: L} \geq \gamma_{2: L} \geq \cdots \geq \gamma_{L_{c}: L}$ are defined as the order statistics of instantaneous SINR, which are obtained by arranging $\left\{\gamma_{l} \mid l=1,2, \ldots, L_{c}\right\}$ in the decreasing order of magnitude. The joint pdf of the order statistics [16], [17] is given by

$$
\begin{aligned}
& p_{\gamma_{1: L}, \gamma_{2: L}, \ldots, \gamma_{L_{c}: L}}\left(\gamma_{1: L}, \gamma_{2: L}, \ldots, \gamma_{L_{c}: L}\right) \\
& =L_{c} ! \cdot\left(\begin{array}{c}
L \\
L_{c}
\end{array}\right) \cdot\left[F_{\gamma_{l}}\left(\gamma_{L_{c}: L}\right)\right]^{L-L_{c}} \cdot \prod_{l=1}^{L_{c}} p_{\gamma_{l}}\left(\gamma_{l: L}\right)
\end{aligned}
$$

where $F(\cdot)$ represents the cdf and $p(\cdot)$ is the relevant pdf.

After the $L_{c}$ strongest paths are selected and combined by a MR-GSC 2-D-RAKE receiver, the total output SINR can be represented as [18]

$$
\gamma_{\mathrm{GSC}}=2 \cdot \sum_{l=1}^{L_{c}} \gamma_{l: L} .
$$

Hence, the BER, conditioned on $\left\{\gamma_{l: L} \mid l=1,2, \ldots, L_{c}\right\}$ can be obtained by

$$
P_{e, \mathrm{GSC}}\left(\gamma_{\mathrm{GSC}}\right)=Q\left(\sqrt{\gamma_{\mathrm{GSC}}}\right) .
$$


Therefore, the resultant BER can be achieved by averaging over the joint pdf of the selected paths in (39) as

$$
\begin{aligned}
P_{e, \mathrm{GSC}}^{\left\langle L_{c}\right\rangle}= & \int_{0}^{\infty} \int_{\gamma_{L_{c}: L}}^{\infty} \cdots \int_{\gamma_{2: L}}^{\infty} Q\left(\sqrt{2 \cdot \sum_{l=1}^{L_{c}} \gamma_{l: L}}\right) \\
& \cdot p_{\gamma_{1: L}, \gamma_{2: L}, \ldots, \gamma_{L_{c}: L}}\left(\gamma_{1: L}, \gamma_{2: L}, \ldots, \gamma_{L_{c}: L}\right) \\
& \cdot d \gamma_{1: L} \cdot d \gamma_{2: L} \cdots d \gamma_{L_{c}: L} .
\end{aligned}
$$

Substituting (37) and (38) into (39) to get the joint pdf and deriving from the general integral formula (42), we further obtain the closed-form BER for several special cases. As $L_{c}=1$, only the strongest one path is selected so that the closed-form BER can be induced as [19]

$$
\begin{aligned}
P_{e, \mathrm{GSC}}^{\langle 1\rangle}= & \left(\begin{array}{c}
L \\
1
\end{array}\right) \cdot \int_{0}^{\infty}\left[F_{\gamma_{l}}\left(\gamma_{1: L}\right)\right]^{L-1} \\
& \cdot p_{\gamma_{l}}\left(\gamma_{1: L}\right) \cdot Q\left(\sqrt{2 \cdot \gamma_{1: L}}\right) \cdot d \gamma_{1: L} \\
= & \frac{L}{2} \cdot \sum_{m=0}^{L-1} \sum_{n=0}^{m}\left(\begin{array}{c}
L-1 \\
m
\end{array}\right) \cdot\left(\begin{array}{c}
m \\
n
\end{array}\right) \\
& \cdot \frac{(-1)^{m}}{(m+1)^{n+2}} \cdot I_{A}\left(n+1, \frac{m+1}{\bar{\gamma}_{c}}\right)
\end{aligned}
$$

where by the definition

$$
I_{A}(p, \alpha)=p ! \cdot\left[1-\sum_{k=0}^{p} \frac{\alpha^{k}}{k ! \cdot \sqrt{\pi}} \cdot \frac{\Gamma(k+1 / 2)}{(1+\alpha)^{k+1 / 2}}\right] .
$$

When $L_{c}=2$ or $L_{c}=3$, the two or three strongest paths are selected and combined so that the resultant BER can be further manipulated through a two- or three-fold integral in (42). The closed-form BER results are derived in [19] and presented in the Appendix.

\section{RECEIVER With IMPERFECT CHANNEL ESTIMATION}

In the downlink, two common pilot signals are transmitted simultaneously from two antennas, which are employed to assist mobile stations to estimate the channel-fading coefficients. The 2-D-RAKE receiver structure is shown in Fig. 1. During the first and second symbol period of the $m$ th STBC block, the pilot channels of the $\hat{l}$ th resolvable path can be despread from (6) and (7) as

$$
\begin{aligned}
r_{p}^{(1)}(m, \hat{l})= & \sum_{n=0}^{N-1} u^{(1)}(m, n) \cdot S_{p}^{*}\left(2 m N+n-\left\lfloor\tau_{\hat{l}} / T_{c}\right\rfloor\right) \\
= & \sqrt{g E_{c}} \cdot N \cdot\left(h_{1, \hat{l}}\right. \\
& \left.+h_{2, \hat{l}}\right)+\sum_{\substack{l=1 \\
l \neq \hat{l}}}^{L} \sqrt{g E_{c}} \cdot R_{p, p}^{(1)}(m ; l, \hat{l}) \cdot\left(h_{1, l}+h_{2, l}\right) \\
& +\sum_{k=1}^{K} \sum_{\substack{l=1 \\
l \neq \hat{l}}}^{L} \sqrt{E_{c}} \cdot R_{d, p}^{(1)}(m, k ; l, \hat{l}) \\
& \cdot\left[b_{1}(k, m) \cdot h_{1, l}-b_{2}(k, m) \cdot h_{2, l}\right]+\eta_{p}^{(1)}(m, \hat{l})
\end{aligned}
$$

$$
\begin{aligned}
r_{p}^{(2)}(m, \hat{l})= & \sum_{n=0}^{N-1} u^{(2)}(m, n) \cdot S_{p}^{*}[(2 m+1) \\
& \left.\cdot N+n-\left\lfloor\tau_{\hat{l}} / T_{c}\right\rfloor\right] \\
= & \sqrt{g E_{c}} \cdot N \cdot\left(h_{1, \hat{l}}-h_{2, \hat{l}}\right) \\
& +\sum_{\substack{l=1 \\
l \neq \hat{l}}}^{L} \sqrt{g E_{c}} \cdot R_{p, p}^{(2)}(m ; l, \hat{l}) \cdot\left(h_{1, l}-h_{2, l}\right) \\
& +\sum_{k=1}^{K} \sum_{\substack{l=1 \\
l \neq \hat{l}}}^{L} \sqrt{E_{c}} \cdot R_{d, p}^{(2)}(m, k ; l, \hat{l}) \\
& \cdot\left[b_{2}(k, m) \cdot h_{1, l}+b_{1}(k, m) \cdot h_{2, l}\right]+\eta_{p}^{(2)}(m, \hat{l})
\end{aligned}
$$

where the background AWGN components $\eta_{p}^{(j)}(m, \hat{l})(j=1,2)$ are defined by

$$
\begin{aligned}
\eta_{p}^{(j)}(m, \hat{l})= & \sum_{n=0}^{N-1} \eta^{(j)}(m, n) \\
& \cdot S_{p}^{*}\left[(2 m+j-1) \cdot N+n-\left\lfloor\tau_{\hat{l}} / T_{c}\right\rfloor\right]
\end{aligned}
$$

The discrete aperiodic correlation function $R_{p, p}^{(j)}(m ; l, \hat{l})$ and $R_{d, p}^{(j)}(m, k ; l, \hat{l})$ are given by (13) with the substitution of the code $S_{d}$ by the corresponding code $S_{p}$ and have the same properties as those of (13).

Considering the $\hat{l}$ th $(\hat{l}=1,2, \ldots, L)$ path of the reference user $k=1$, the data channel is despread during the first and second symbol period of the $m$ th STBC block as

$$
\begin{aligned}
\hat{r}_{d}^{(1)}(m, \hat{l})= & \sum_{n=0}^{N-1} u^{(1)}(m, n) \\
& \cdot S_{d}^{*}\left(1,2 m N+n-\left\lfloor\tau_{\hat{l}} / T_{c}\right\rfloor\right) \\
= & r_{d}^{(1)}(m, \hat{l})+\sum_{\substack{l=1 \\
l \neq l}}^{L} \sqrt{g E_{c}} \\
& \cdot R_{p, d}^{(1)}(m ; l, \hat{l}) \cdot\left(h_{1, l}+h_{2, l}\right) \\
\hat{r}_{d}^{(2)}(m, \hat{l})= & \sum_{n=0}^{N-1} u^{(2)}(m, n) \\
& \cdot S_{d}^{*}\left[1,(2 m+1) \cdot N+n-\left\lfloor\tau_{\hat{l}} / T_{c}\right\rfloor\right] \\
= & r_{d}^{(2)}(m, \hat{l})+\sum_{\substack{l=1 \\
l \neq l}}^{L} \sqrt{g E_{c}} \\
& \cdot R_{p, d}^{(2)}(m ; l, \hat{l}) \cdot\left(h_{1, l}-h_{2, l}\right)
\end{aligned}
$$

where $r_{d}^{(j)}(m, \hat{l})(j=1,2)$ are given in (10) and (11). By comparing (48) and (49) with (10) and (11), it is seen that the despread data channel signals suffer from one more interference term due to the pilot signals. $R_{p, d}^{(j)}(m ; l, \hat{l})$ is given by (13) withthe substitution of $S_{d}$ by $S_{p}$ and has the same statistic 
properties. Therefore, conditioned on the channel-fading coefficients at the $\hat{l}$ th path in the $m$ th STBC coding block and the data bits, the mean and variance of $\hat{r}_{d}^{(j)}(m, \hat{l})(j=1,2)$ are given by

$$
\begin{aligned}
E\left\{\hat{r}_{d}^{(1)}(m, \hat{l})\right\}= & \sqrt{E_{c}} \cdot N \cdot\left[b_{1}(1, m) \cdot h_{1, \hat{l}}-b_{2}(1, m) \cdot h_{2, \hat{l}}\right] \\
E\left\{\hat{r}_{d}^{(2)}(m, \hat{l})\right\}= & \sqrt{E_{c}} \cdot N \cdot\left[b_{2}(1, m) \cdot h_{1, \hat{l}}+b_{1}(1, m) \cdot h_{2, \hat{l}}\right] \\
\operatorname{Var}\left\{\hat{r}_{d}^{(j)}(m, \hat{l})\right\}=E & \left\{\left[\hat{r}_{d}^{(j)}(m, \hat{l})-E\left\{\hat{r}_{d}^{(j)}(m, \hat{l})\right\}\right]\right. \\
& \left.\cdot\left[\hat{r}_{d}^{(j)}(m, \hat{l})-E\left\{\hat{r}_{d}^{(j)}(m, \hat{l})\right\}\right]^{*}\right\} \\
= & E_{c} \cdot N \cdot(g+K) \cdot(L-1) \cdot 4 \sigma^{2}+N \cdot \frac{\eta_{0}}{2} .
\end{aligned}
$$

Based on the despread pilot signals in (45) and (46), the initial estimates of pair of channels between two transmit antennas and receive antenna at the $\hat{l}$ th resolvable path in the $m$ th STBC block can be manipulated as

$$
\begin{aligned}
& \hat{r}_{p}^{(1)}(m, \hat{l})=\frac{1}{2 N \cdot \sqrt{g E_{c}}} \cdot\left[r_{p}^{(1)}(m, \hat{l})+r_{p}^{(2)}(m, \hat{l})\right] \\
& =h_{1, \hat{l}}+\frac{1}{2 N \cdot \sqrt{g E_{c}}} \cdot\left[\eta_{p}^{(1)}(m, \hat{l})+\eta_{p}^{(2)}(m, \hat{l})\right] \\
& +\frac{1}{2 N} \cdot \sum_{\substack{l=1 \\
l \neq \hat{l}}}^{L}\left[R_{p, p}^{(1)}(m ; l, \hat{l}) \cdot\left(h_{1, l}+h_{2, l}\right)\right. \\
& \left.+R_{p, p}^{(2)}(m ; l, \hat{l}) \cdot\left(h_{1, l}-h_{2, l}\right)\right] \\
& +\frac{1}{2 N \cdot \sqrt{g}} \cdot \sum_{k=1}^{K} \sum_{\substack{l=1 \\
l \neq \hat{l}}}^{L}\left\{R_{d, p}^{(1)}(m, k ; l, \hat{l})\right. \\
& \cdot\left[b_{1}(k, m) \cdot h_{1, l}-b_{2}(k, m) \cdot h_{2, l}\right] \\
& +R_{d, p}^{(2)}(m, k ; l, \hat{l}) \\
& \left.\cdot\left[b_{2}(k, m) \cdot h_{1, l}+b_{1}(k, m) \cdot h_{2, l}\right]\right\} \\
& \hat{r}_{p}^{(2)}(m, \hat{l})=\frac{1}{2 N \cdot \sqrt{g E_{c}}} \cdot\left[r_{p}^{(1)}(m, \hat{l})-r_{p}^{(2)}(m, \hat{l})\right] \\
& =h_{2, \hat{l}}+\frac{1}{2 N \cdot \sqrt{g E_{c}}} \cdot\left[\eta_{p}^{(1)}(m, \hat{l})-\eta_{p}^{(2)}(m, \hat{l})\right] \\
& +\frac{1}{2 N} \cdot \sum_{\substack{l=1 \\
l \neq \hat{l}}}^{L}\left[R_{p, p}^{(1)}(m ; l, \hat{l}) \cdot\left(h_{1, l}+h_{2, l}\right)\right. \\
& \left.-R_{p, p}^{(2)}(m ; l, \hat{l}) \cdot\left(h_{1, l}-h_{2, l}\right)\right] \\
& +\frac{1}{2 N \cdot \sqrt{g}} \cdot \sum_{k=1}^{K} \sum_{\substack{l=1 \\
l \neq \hat{l}}}^{L}\left\{R_{d, p}^{(1)}(m, k ; l, \hat{l})\right. \\
& \text { - }\left[b_{1}(k, m) \cdot h_{1, l}-b_{2}(k, m) \cdot h_{2, l}\right] \\
& \text { - } R_{d, p}^{(2)}(m, k ; l, \hat{l}) \\
& \left.\cdot\left[b_{2}(k, m) \cdot h_{1, l}+b_{1}(k, m) \cdot h_{2, l}\right]\right\} \text {. }
\end{aligned}
$$

Similarly, conditioned on the channel-fading coefficients at the $\hat{l}$ th path in the $m$ th STBC coding block, the initial estimates $\hat{r}_{p}^{(i)}(m, \hat{l})(i=1,2)$ have the same variance, given by

$$
\begin{aligned}
\operatorname{Var}\left\{\hat{r}_{p}^{(i)}(m, \hat{l})\right\}= & E\left\{\left[\hat{r}_{p}^{(i)}(m, \hat{l})-E\left\{\hat{r}_{p}^{(i)}(m, \hat{l})\right\}\right]\right. \\
& \left.\cdot\left[\hat{r}_{p}^{(i)}(m, \hat{l})-E\left\{\hat{r}_{p}^{(i)}(m, \hat{l})\right\}\right]^{*}\right\} \\
= & \frac{1}{g N} \cdot(g+K) \cdot(L-1) \\
& \cdot 2 \sigma^{2}+\frac{1}{2 g N \cdot E_{c}} \cdot \frac{\eta_{0}}{2} .
\end{aligned}
$$

Note that the above initial channel estimates with the assistance of pilot transmission suffer not only from the background AWGN, but also from the multiple access and multipath interference (MPI) resulting from data channels of all simultaneous active users as well as two pilot channels. It is seen that the channel estimation under TD-STBC is deteriorated by more MPI than that in conventional DS-CDMA system with only one transmit antenna. Therefore, it is vital to exploit some techniques to estimate channels more accurately so that the performance of TD-STBC can be improved significantly.

For the slow-fading channel, it is assumed that the channel coefficients $h_{i, l}$ remain invariable during several consecutive STBC blocks; thus, a $N_{P}$-tap low-pass filter (LPF) can be used to improve the accuracy of the pilot-assisted channel estimation. In other words, the improved channel estimation can be obtained through time averaging

$$
\hat{h}_{i, l}(m)=\frac{1}{N_{P}} \cdot \sum_{m \in \Theta} \hat{r}_{p}^{(i)}(m, \hat{l})
$$

where any one of example sets given below might be adopted as the timing-average window

$$
\begin{aligned}
& \Theta \equiv\left\{m-N_{P}+1, m\right\} \\
& \Theta \equiv\left\{m-\left\lfloor\frac{N_{P}-1}{2}\right\rfloor, m+\left\lfloor\frac{N_{P}}{2}\right\rfloor\right\} .
\end{aligned}
$$

Assuming that the noise and interference terms in successive $\hat{r}_{p}^{(i)}(m, \hat{l})$ with different $m$ are independent from each other, one obtains

$$
\begin{gathered}
E\left\{\hat{h}_{i, l}(m)\right\}=E\left\{\hat{r}_{p}^{(i)}(m, \hat{l})\right\}=h_{i, \hat{l}} \\
\operatorname{Var}\left\{\hat{h}_{i, l}(m)\right\}=\frac{1}{N_{P}} \cdot \operatorname{Var}\left\{\hat{r}_{p}^{(i)}(m, \hat{l})\right\} .
\end{gathered}
$$

With respect to the $\hat{l}_{\text {th }}$ resolvable path, the branch random variables of two estimated data bits of the first user in the $m$ th STBC block can be constructed through STBC decoding as

$$
\begin{aligned}
& \hat{d}_{1}(m, \hat{l})=\hat{r}_{d}^{(1)}(m, \hat{l}) \cdot \hat{h}_{1, \hat{l}}^{*}(m)+\hat{r}_{d}^{(2)^{*}}(m, \hat{l}) \cdot \hat{h}_{2, \hat{l}}(m) \\
& \hat{d}_{2}(m, \hat{l})=\hat{r}_{d}^{(2)}(m, \hat{l}) \cdot \hat{h}_{1, \hat{l}}^{*}(m)-\hat{r}_{d}^{(1)^{*}}(m, \hat{l}) \cdot \hat{h}_{2, \hat{l}}(m) .
\end{aligned}
$$

Since the pair of channel coefficients $h_{1, \hat{l}}$ and $h_{2, \hat{l}}$ are mutually independent and the discrete aperiodic correlation functions of spreading code sequences are independent random variables, 
all of $\hat{r}_{d}^{(1)}(m, \hat{l}), \hat{r}_{d}^{(2)}(m, \hat{l}), \hat{h}_{1, \hat{l}}(m)$, and $\hat{h}_{2, \hat{l}}(m)$ are independent from each other. Moreover, the two-branch random variables of estimated data bits $\hat{d}_{j}(m, \hat{l})(j=1,2)$ are the sum of many independent random variables and can be approximated as Gaussian variables, conditioned on the channel fading coefficients of the $\hat{l}$ th path $h_{i, \hat{l}}(i=1,2)$ and the data bit $b_{1}(1, m)$. The conditional mean of $\hat{d}_{1}(m, \hat{l})$ is given by

$$
\begin{aligned}
E\left\{\hat{d}_{1}(m, \hat{l})\right\}= & E\left\{\hat{r}_{d}^{(1)}(m, \hat{l})\right\} \cdot E\left\{\hat{h}_{1, \hat{l}}^{*}(m)\right\} \\
& +E\left\{\hat{r}_{d}^{(2)^{*}}(m, \hat{l})\right\} \cdot E\left\{\hat{h}_{2, \hat{l}}(m)\right\} \\
= & \sqrt{E_{c}} \cdot N \cdot b_{1}(1, m) \cdot\left(\left|h_{1, \hat{l}}\right|^{2}+\left|h_{2, \hat{l}}\right|^{2}\right) .
\end{aligned}
$$

Meanwhile, the conditional variance of $\hat{d}_{1}(m, \hat{l})$ can be obtained as

$$
\begin{aligned}
\operatorname{Var}\{ & \left.\hat{d}_{1}(m, \hat{l})\right\} \\
= & \left|E\left\{\hat{r}_{d}^{(1)}(m, \hat{l})\right\}\right|^{2} \cdot \operatorname{Var}\left\{\hat{h}_{1, \hat{l}}^{*}(m)\right\} \\
& +\left|E\left\{\hat{h}_{1, \hat{l}}^{*}(m)\right\}\right|^{2} \cdot \operatorname{Var}\left\{\hat{r}_{d}^{(1)}(m, \hat{l})\right\} \\
& +\operatorname{Var}\left\{\hat{r}_{d}^{(1)}(m, \hat{l})\right\} \cdot \operatorname{Var}\left\{\hat{h}_{1, \hat{l}}^{*}(m)\right\} \\
& +\left|E\left\{\hat{r}_{d}^{(2) *}(m, \hat{l})\right\}\right|^{2} \cdot \operatorname{Var}\left\{\hat{h}_{2, \hat{l}}(m)\right\} \\
& +\left|E\left\{\hat{h}_{2, \hat{l}}(m)\right\}\right|^{2} \cdot \operatorname{Var}\left\{\hat{r}_{d}^{(2) *}(m, \hat{l})\right\} \\
& +\operatorname{Var}\left\{\hat{r}_{d}^{(2) *}(m, \hat{l})\right\} \cdot \operatorname{Var}\left\{\hat{h}_{2, \hat{l}}(m)\right\} \\
= & N \cdot\left(1+\frac{1}{g N_{P}}\right) \cdot\left[E_{c} \cdot(g+K)\right. \\
& \left.\cdot(L-1) \cdot 4 \sigma^{2}+\frac{\eta_{0}}{2}\right] \cdot\left(\left|h_{1, \hat{l}}\right|^{2}+\left|h_{2, \hat{l}}\right|^{2}\right) \\
& +\frac{1}{g N_{P} \cdot E_{c}} \cdot\left[E_{c} \cdot(g+K)\right. \\
& \left.\cdot(L-1) \cdot 4 \sigma^{2}+\frac{\eta_{0}}{2}\right]^{2} \cdot
\end{aligned}
$$

Similarly, the conditional statistics of $\hat{d}_{2}(m, \hat{l})$ can be derived.

\section{A. Maximal Ratio-Equal Gain Combiner}

After the despreading and STBC decoding at selected paths, a 2-D-RAKE MR-EGC receiver is utilized to combine the signals from the first $L_{c}$ arriving paths. Under the assumption of mutually independent channel fading along multiple paths, the decision variables in different branches of 2-D-RAKE receiver are independent from each other and the output of the combiner can be represented as

$$
\hat{d}_{j}(m)=\sum_{l=1}^{L_{c}} \hat{d}_{j}(m, l) .
$$

Thus, the decision variable $\hat{d}_{j}(m)(j=1,2)$ is a Gaussian variable with conditional mean and variance given by

$$
\begin{aligned}
E\left\{\hat{d}_{j}(m)\right\} & =\sum_{l=1}^{L_{c}} E\left\{\hat{d}_{j}(m, l)\right\} \\
& =\sqrt{E_{c}} \cdot N \cdot b_{j}(1, m) \cdot \zeta
\end{aligned}
$$

$$
\begin{aligned}
\operatorname{Var}\left\{\hat{d}_{j}(m)\right\}= & \sum_{l=1}^{L_{c}} \operatorname{Var}\left\{\hat{d}_{j}(m, l)\right\} \\
= & N \cdot\left(1+\frac{1}{g N_{P}}\right) \\
& \cdot\left[E_{c} \cdot(g+K) \cdot(L-1) \cdot 4 \sigma^{2}+\frac{\eta_{0}}{2}\right] \cdot \zeta \\
& +\frac{L_{c}}{g N_{P} \cdot E_{c}} \\
& \cdot\left[E_{c} \cdot(g+K) \cdot(L-1) \cdot 4 \sigma^{2}+\frac{\eta_{0}}{2}\right]^{2}
\end{aligned}
$$

where $\zeta$ is defined as the same as that in (26). It is seen that the variance in (67) will reduce to the expression of (25) when the parameter $N_{P} \rightarrow \infty$ and $g \ll K$. In other words, when the number of taps of the LPF trends to be infinite, perfect channel estimation is roughly achieved under the assumption of static channels. Therefore, the system performance will approximate to the results given in Section III.

Furthermore, conditioned on the instantaneous fading channel amplitudes of the selected paths, the BER is the same for $\hat{d}_{j}(m)(j=1,2)$ and given by

$$
\begin{aligned}
\hat{P}_{e, \mathrm{EGC}}(\hat{\zeta}) & =Q\left(\left\{\frac{\left|E\left\{\hat{d}_{j}(m)\right\}\right|^{2}}{\operatorname{Var}\left\{\hat{d}_{j}(m)\right\}}\right\}^{1 / 2}\right) \\
& =Q\left[\left(\frac{\hat{\zeta}^{2}}{\mathrm{~A} \cdot \hat{\zeta}+L_{c} \cdot \mathrm{B}}\right)^{1 / 2}\right]
\end{aligned}
$$

where, by the definitions

$$
\begin{aligned}
\hat{\zeta}= & \frac{1}{2 \sigma^{2}} \cdot \sum_{l=1}^{L_{c}}\left(\left|h_{1, l}\right|^{2}+\left|h_{2, l}\right|^{2}\right) \\
\mathrm{A}= & \left(\frac{1}{N}+\frac{1}{g N_{P} \cdot N}\right) \\
& \cdot\left[2 \cdot(g+K) \cdot(L-1)+\frac{N}{2 \bar{\gamma}_{b}}\right] \\
\mathrm{B}= & \frac{1}{g N_{P} \cdot N^{2}} \cdot\left[2 \cdot(g+K) \cdot(L-1)+\frac{N}{2 \bar{\gamma}_{b}}\right]^{2} .
\end{aligned}
$$

Since the channel fading coefficients $h_{i, l}(m)$ are assumed as i.i.d. complex Gaussian distributed random variables, the pdf of $\hat{\zeta}$ is given by

$$
p_{\hat{\zeta}}(\hat{\zeta})=\frac{1}{\Gamma\left(2 L_{c}\right)} \cdot \hat{\zeta}^{2 L_{c}-1} \cdot e^{-\hat{\zeta}}
$$

Therefore, the resultant BER can be obtained by averaging the conditional probability $\hat{P}_{e, \mathrm{EGC}}(\hat{\zeta})$ in (68) over the pdf of $\hat{\zeta}$ in (72), i.e.,

$$
\begin{aligned}
\hat{P}_{e, \mathrm{EGC}}= & \int_{0}^{\infty} \hat{P}_{e, \mathrm{EGC}}(\hat{\zeta}) \cdot p_{\hat{\zeta}}(\hat{\zeta}) \cdot d \hat{\zeta} \\
= & \int_{0}^{\infty} Q\left[\left(\frac{\hat{\zeta}^{2}}{\mathrm{~A} \cdot \hat{\zeta}+L_{c} \cdot \mathrm{B}}\right)^{1 / 2}\right] \\
& \cdot p_{\hat{\zeta}}(\hat{\zeta}) \cdot d \hat{\zeta} \\
= & \frac{1}{\Gamma\left(2 L_{c}\right)} \cdot \int_{0}^{\infty} Q\left(\sqrt{\frac{y^{2}}{\mathrm{~A} \cdot y+L_{c} \cdot \mathrm{B}}}\right) \\
& \cdot y^{2 L_{c}-1} \cdot e^{-y} \cdot d y .
\end{aligned}
$$


Note that for a given SNR and number of active users, the parameter of power ratio $g$ might impact the system performance significantly. When $g$ is very large, the pilot channel signal might introduce severe multipath interference to all active users. On the other hand, when $g$ is very small, the channel-estimation quality becomes poor, so that the BER degrades. Therefore, there exists an optimal value of power ratio $g$ that would maximize the SINR or minimize the BER under certain scenarios, such as given average transmit power and the number of the active users. By setting $\partial\left(\mathrm{A} \cdot \hat{\zeta}+L_{c} \cdot \mathrm{B}\right) / \partial g=0$, conditioned on $\hat{\zeta}$, the optimal $g$ is given by

$$
g_{\text {opt }}(\hat{\zeta})=\sqrt{\frac{a_{1} \cdot \hat{\zeta}+b_{1}}{a_{2} \cdot \hat{\zeta}+b_{2}}}
$$

where, by definitions

$$
\begin{aligned}
a_{1}= & 8 K \cdot(L-1) \cdot N+2 N^{2} \cdot \bar{\gamma}_{b}^{-1} \\
b_{1}= & L_{c} \cdot\left[8 K \cdot N \cdot(L-1) \cdot \bar{\gamma}_{b}^{-1}\right. \\
& \left.+N^{2} \cdot \bar{\gamma}_{b}^{-2}+16 K^{2} \cdot(L-1)^{2}\right] \\
a_{2}= & 8 \cdot(L-1) \cdot N_{P} \cdot N \\
b_{2}= & 16 L_{c} \cdot(L-1)^{2} .
\end{aligned}
$$

Similarly, the average $g_{\text {opt }}$ can be obtained by averaging the conditional $g_{\text {opt }}(\hat{\zeta})$ in (74) over the pdf of $\hat{\zeta}$ in (72) [13], i.e.,

$$
\begin{aligned}
g_{\mathrm{opt}}= & \int_{0}^{\infty} g_{\mathrm{opt}}(\hat{\zeta}) \cdot p_{\hat{\zeta}}(\hat{\zeta}) \cdot d \hat{\zeta} \\
= & \frac{1}{\Gamma\left(2 L_{c}\right)} \cdot \int_{0}^{\infty} \hat{\zeta}^{2 L_{c}-1} \\
& \cdot \sqrt{\frac{a_{1} \cdot \hat{\zeta}+b_{1}}{a_{2} \cdot \hat{\zeta}+b_{2}}} \cdot e^{-\hat{\zeta}} \cdot d \hat{\zeta} .
\end{aligned}
$$

\section{B. Maximal Ratio-Generalized Selection Combiner}

Noted that $\hat{d}_{j}(m, l)(j=1,2)$ have the same SINR property and based on (63) and (64), the instantaneous SINR of the branch random variables $\hat{d}_{1}(m, l)$ in the $l$ th $(l=1,2, \ldots, L)$ path is derived as

$$
\hat{\gamma}_{l}=\frac{\left|E\left\{\hat{d}_{1}(m, l)\right\}\right|^{2}}{\operatorname{Var}\left\{\hat{d}_{1}(m, l)\right\}}=\frac{\hat{\zeta}_{l}^{2}}{\mathrm{~A} \cdot \hat{\zeta}_{l}+\mathrm{B}}
$$

where, by the definition

$$
\hat{\zeta}_{l}=\frac{1}{2 \sigma^{2}} \cdot\left(\left|h_{1, l}\right|^{2}+\left|h_{2, l}\right|^{2}\right) .
$$

It can be proven that the variable $\hat{\gamma}_{l}$ in (80) is the monotonous increasing function of the variable $\hat{\zeta}_{l}$ in (81). Therefore, selecting the paths with highest instantaneous value of $\hat{\zeta}_{l}$ is equivalent to selecting the paths with highest instantaneous SINR $\hat{\gamma}_{l}$. Moreover, $\hat{\zeta}_{1: L} \geq \hat{\zeta}_{2: L} \geq \cdots \geq \hat{\zeta}_{L: L}$ are defined as the order statistic of variables, obtained by arranging $\left\{\hat{\zeta}_{l} \mid l=1,2, \ldots, L\right\}$ in the decreasing order of magnitude. The joint pdf of the order statistic variables $\hat{\zeta}_{l: L}$ is given by

$$
\begin{aligned}
& p_{\hat{\zeta}_{1: L}, \hat{\zeta}_{2: L}, \ldots, \hat{\zeta}_{L_{c}: L}}\left(\hat{\zeta}_{1: L}, \hat{\zeta}_{2: L}, \ldots, \hat{\zeta}_{L_{c}: L}\right) \\
& \quad=L_{c} ! \cdot\left(\begin{array}{c}
L \\
L_{c}
\end{array}\right) \cdot\left[F_{\hat{\zeta}_{l}}\left(\hat{\zeta}_{L_{c}: L}\right)\right]^{L-L_{c}} \cdot \prod_{l=1}^{L_{c}} p_{\hat{\zeta}_{l}}\left(\hat{\zeta}_{l: L}\right)
\end{aligned}
$$

where the pdf and cdf of $\hat{\zeta}_{l}$ are given by

$$
\begin{aligned}
& p_{\hat{\zeta}_{l}}\left(\hat{\zeta}_{l}\right)=\hat{\zeta}_{l} \cdot \exp \left(-\hat{\zeta}_{l}\right) \\
& F_{\hat{\zeta}_{l}}\left(\hat{\zeta}_{l}\right)=1-\left(1+\hat{\zeta}_{l}\right) \cdot \exp \left(-\hat{\zeta}_{l}\right) .
\end{aligned}
$$

Thus, the total output SINR of the 2-D-RAKE MR-GSC receiver that selects and combines $L_{c}$ strongest paths can be presented as

$$
\hat{\gamma}_{\mathrm{GSC}}=\frac{\left(\sum_{l=1}^{L_{c}} \hat{\zeta}_{l: L}\right)^{2}}{A \cdot \sum_{l=1}^{L_{c}} \hat{\zeta}_{l: L}+L_{c} \cdot B}
$$

and the BER, conditioned on $\left\{\hat{\zeta}_{l: L} \mid l=1,2, \ldots, L_{c}\right\}$, is given by

$$
\hat{P}_{e, \mathrm{GSC}}\left(\hat{\gamma}_{\mathrm{GSC}}\right)=Q\left(\sqrt{\hat{\gamma}_{\mathrm{GSC}}}\right) .
$$

Then, the average BER can be obtained by averaging (86) over the joint pdf of the selected fading paths in (82) as

$$
\begin{aligned}
\hat{P}_{e, \mathrm{GSC}}^{\left\langle L_{c}\right\rangle}= & \int_{0}^{\infty} \int_{\hat{\zeta}_{L_{c}: L}}^{\infty} \cdots \int_{\hat{\zeta}_{2: L}}^{\infty} \hat{P}_{e, \mathrm{GSC}}\left(\hat{\gamma}_{\mathrm{GSC}}\right) \\
& \cdot p_{\hat{\zeta}_{1: L}, \hat{\zeta}_{2: L}, \ldots, \hat{\zeta}_{L_{c}: L}}\left(\hat{\zeta}_{1: L}, \hat{\zeta}_{2: L}, \ldots, \hat{\zeta}_{L_{c}: L}\right) \\
& \cdot d \hat{\zeta}_{1: L} \cdot d \hat{\zeta}_{2: L} \cdots d \hat{\zeta}_{L_{c}: L}
\end{aligned}
$$

\section{NUMERICAL RESUltS}

In this section, the effects of different system configurations and parameters on the BER performance of TD-STBC are numerically evaluated. In order to show the spatial and path diversity gain provided by the 2-D-RAKE receiver, the total transmit power is restricted as constant, irrespective of the number of transmit antennas and resolvable multipaths. Therefore, the parameter of SNR per bit per antenna per path $\bar{\gamma}_{p}$ in (34) is used to calculate BER while the SNR per bit $\bar{\gamma}_{b}$ in (33) is used to plot the performance figures. Unless otherwise noted, the number of resolvable multipaths $L=6$, the number of active users $K=10$, and the spreading factor $N=128$, the power ratio of the pilot to data channel $g=3 \mathrm{~dB}$ and the number of taps of LPF adopted $N_{P}=4$.

In Fig. 2, the BER of the 2-D-RAKE MR-EGC receiver is illustrated versus the average SNR per bit $\bar{\gamma}_{b}$ for a different number of RAKE fingers, i.e., $L_{c}=1,3,6$, respectively. For comparison, the BER results of imperfect channel estimation and ideal CSI are shown. It is clearly seen that the RAKE receiver improves the performance for both imperfect channel estimation and ideal CSI when the number of fingers increases. However, the improvement in BER for imperfect channel estimation is not as much as that for ideal CSI. A similar trend in BER can be seen and the same conclusion can be obtained from Fig. 3 when MR-GSC is used, instead of MR-EGC. In order to clearly show the difference of BER performance between MR-EGC and MR-GSC, the two idle curves $\left(L_{c}=3\right)$ from both Figs. 2 and 3 are placed into Fig. 4. It can be seen that MR-GSC outperforms MR-EGC. However, the cost of this outperformance is more implementation complexity. 


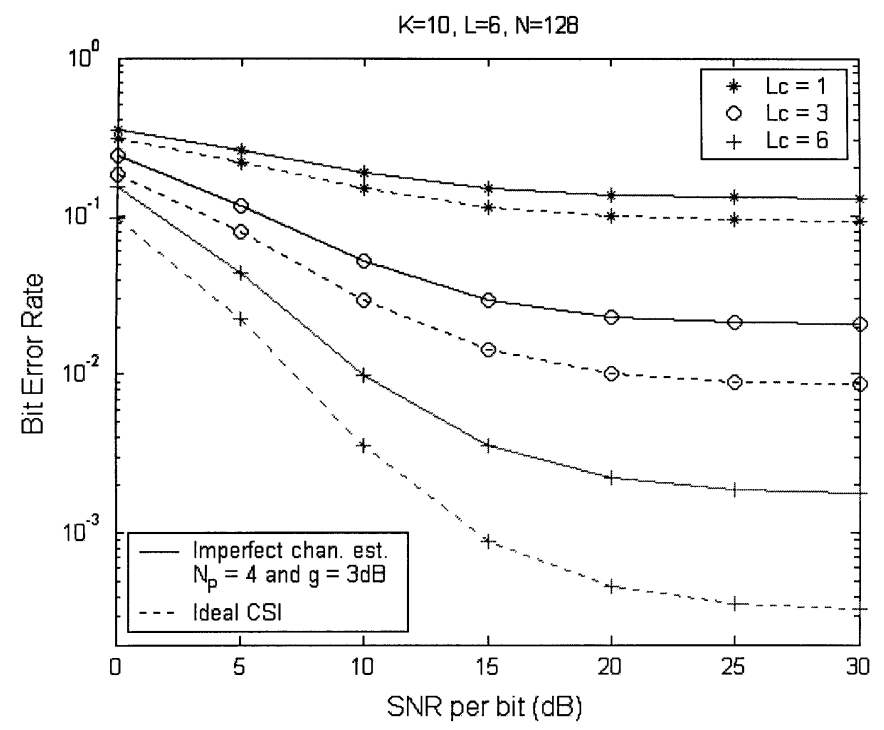

Fig. 2. BER of the 2-D-RAKE MR-EGC receiver.

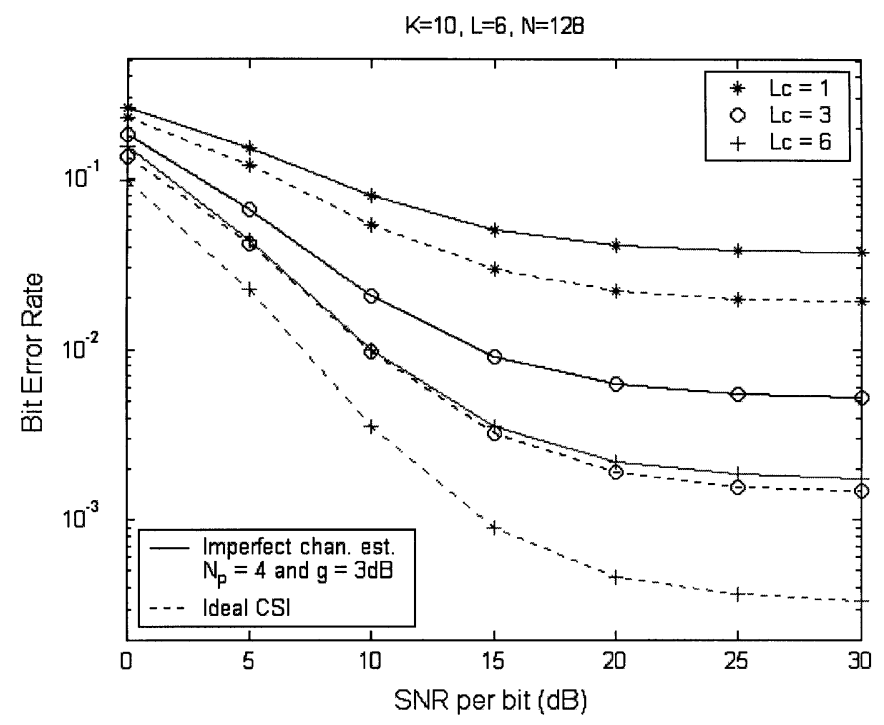

Fig. 3. BER of the 2-D-RAKE MR-GSC receiver.

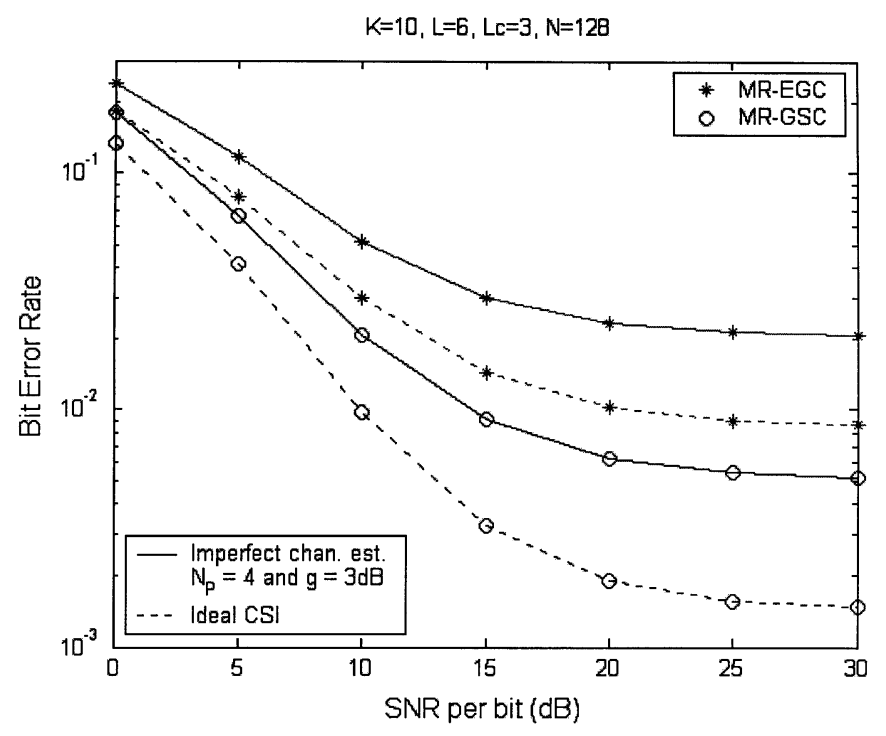

Fig. 4. BER comparison of the MR-EGC and MR-GSC receiver with $L_{c}=3$.

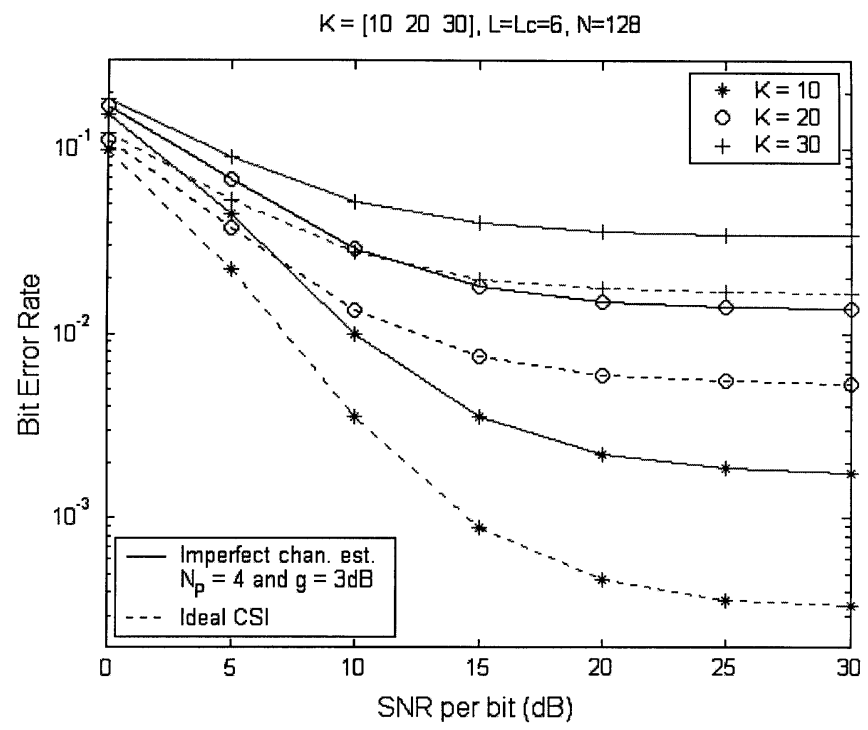

Fig. 5. BER of full 2-D-RAKE MR-GSC/EGC receiver with a different number of users.

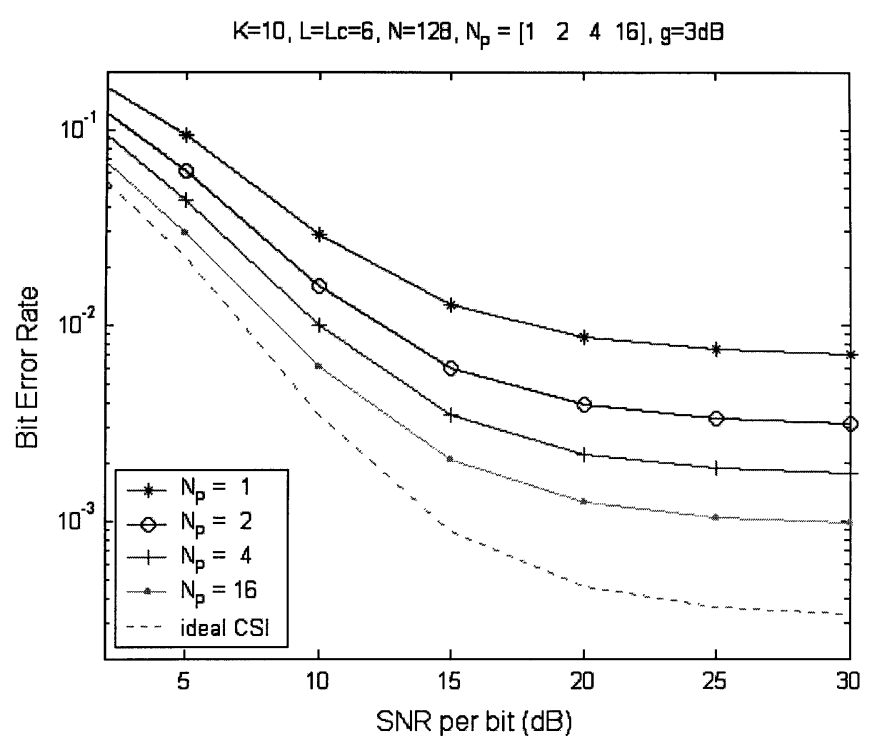

Fig. 6. BER of the full 2-D-RAKE receiver with different quality of channel estimation.

Fig. 5 directly shows the BER performance of full (i.e., $L_{c}=$ $L=6)$ MR-GSC or MR-EGC 2-D-RAKE receivers under perfect and imperfect channel estimation for a different number of active users. It can be seen that the gap in BER between the imperfect channel estimation and ideal CSI decreases when the number of users increases. However, the required SNR to achieve a desired BER actually increases with $K$; therefore, the accurate channel estimation becomes increasingly more important as more users are involved.

Fig. 6 shows the BER of full (i.e., $L_{c}=L=6$ ) MR-GSC or MR-EGC 2-D-RAKE receiver for various numbers of taps $N_{P}=1,2,4$, and 16 , respectively. It can be seen that the system performance can be improved significantly when $N_{P}$ increases in static (or slow) fading channels. This is because increasing $N_{P}$ means improving the quality of the channel estimation. 


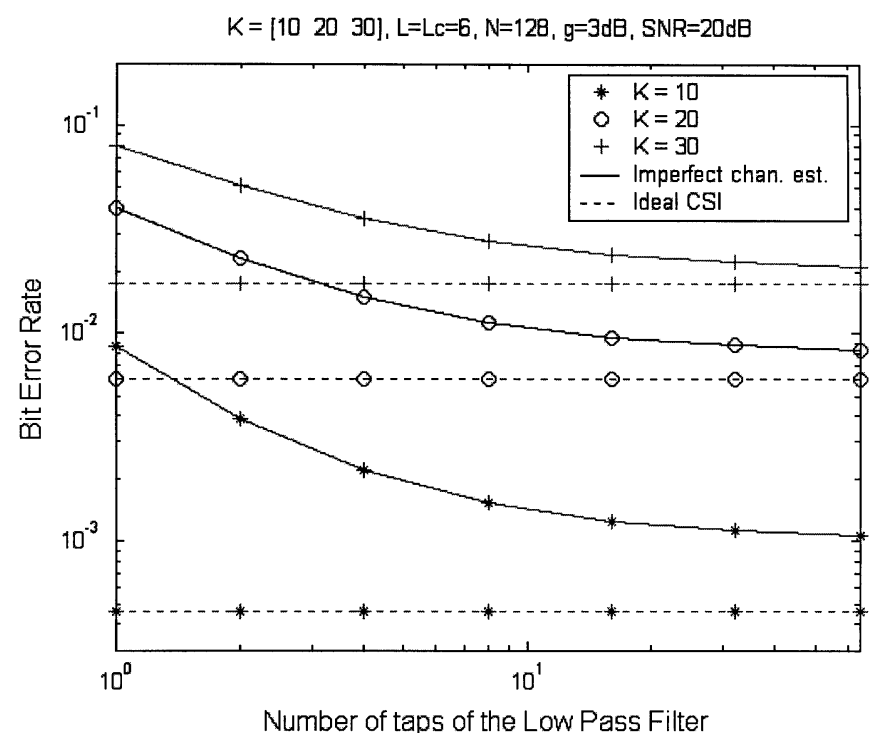

Fig. 7. BER of the full 2-D-RAKE receiver versus the number of taps of LPF with a different number of users.

$K=\left[\begin{array}{llll}5 & 10 & 20 & 30\end{array}\right], L=L C=6, N=128, N_{P}=4, S N R=20 d B$

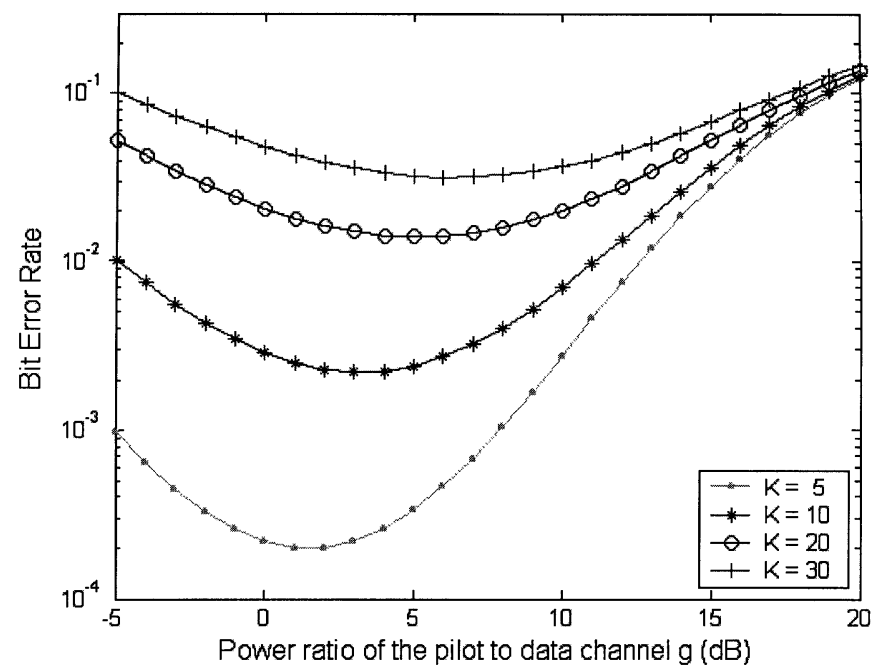

Fig. 8. BER of the full 2-D-RAKE receiver versus the power ratio $g$ with a different number of users.

Fig. 7 shows the BER of the full 2-D-RAKE receiver versus the number of taps of LPF with different number of users when the power ratio of pilot to data channel is $g=3 \mathrm{~dB}$ and the average SNR $\bar{\gamma}_{b}=20 \mathrm{~dB}$. It can be seen that for a given $K$, when $N_{P}$ increases from a small number, the performance of imperfect channel estimation improves significantly. However, further increasing $N_{P}$ only improves performance slightly. For example, increasing $N_{P}$ beyond 20 improves BER performance insignificantly.

Fig. 8 illustrates the BER of the full 2-D-RAKE receiver versus the power ratio $g$ with a different number of active users. The number of taps of LPF is $N_{P}=4$ and $\bar{\gamma}_{b}=20 \mathrm{~dB}$. It can be observed that an optimal power ratio $g$ exists for a given $K$ and increases as the number of active users increases. This is because, when the number of users is large, the multiple access and multipath interference increases for pilot. In order to maintain the quality of channel estimates, more pilot power is needed. For $K=10$, the optimal power ratio $g$ is around $3 \mathrm{~dB}$. This is

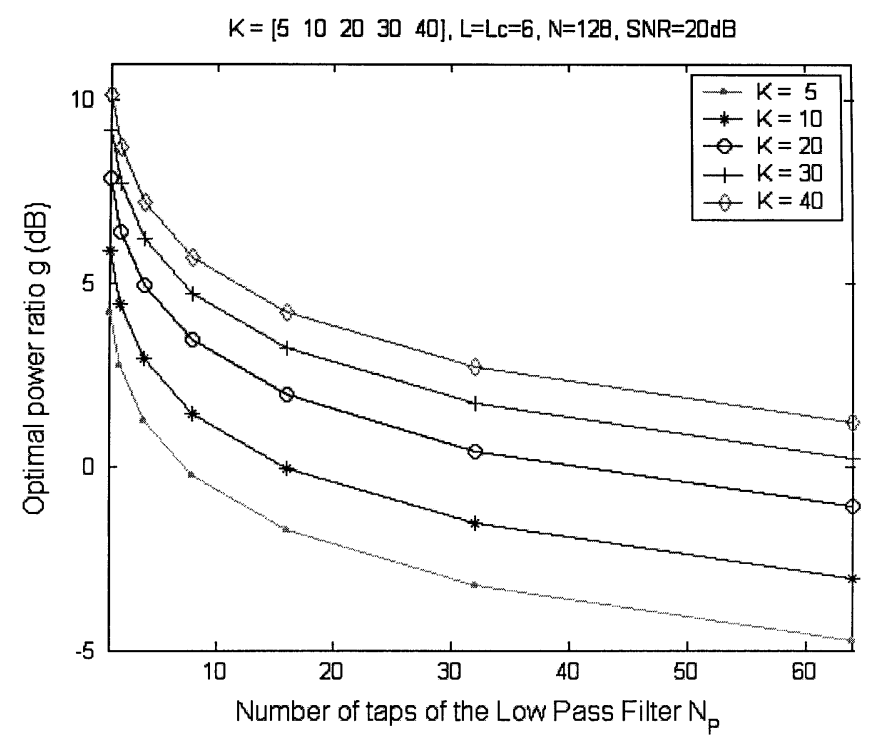

Fig. 9. Optimal power ratio $g_{\text {opt }}$ versus the number of taps of LPF for the full 2-D-RAKE receiver.

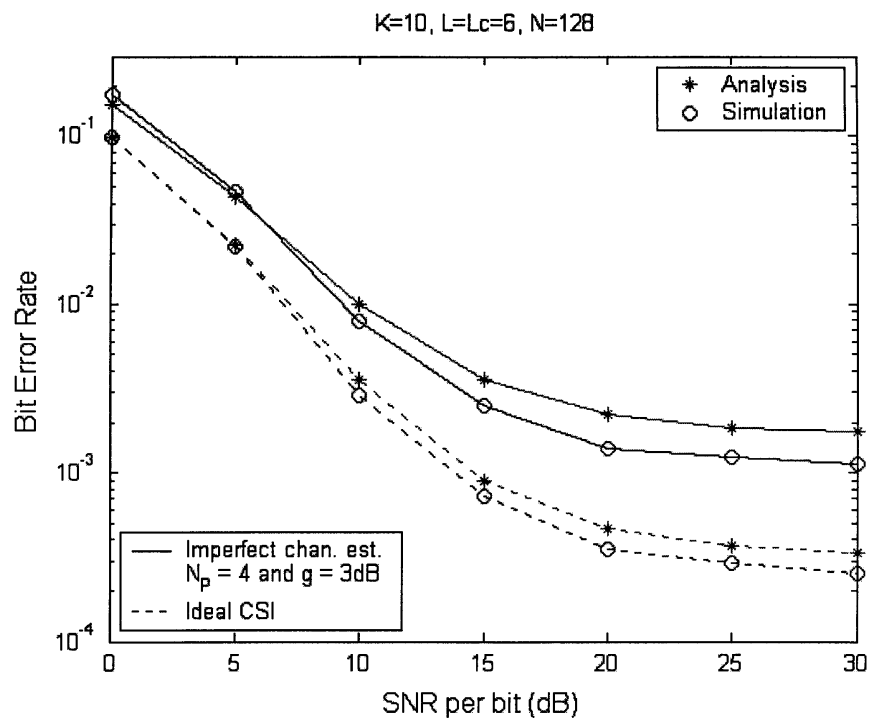

Fig. 10. Comparison of the analysis and simulation results of the full 2-D-RAKE receiver.

consistent with the optimal ratio $g_{\text {opt }}$ derived by (79), which is shown in Fig. 9. This implies that the BS should dynamically adjust the power ratio of the pilot to data channels according to the number of total active system users.

In Fig. 9, for the full 2-D-RAKE receiver the average optimal power ratio $g_{\mathrm{opt}}$ defined in (79) is plotted versus the number of the taps of LPF with a different number of active users. Again, the average SNR is large, i.e., $\bar{\gamma}_{b}=20 \mathrm{~dB}$. It can be seen that the optimal value $g_{\text {opt }}$ decreases monotonously as the number of taps of LPF increases. This is because increasing $N_{P}$ can improve the channel estimation so that the transmission power of the pilot signal can be proportionally reduced to maintain a given channel-estimation quality. Moreover, the optimal ratio $g_{\text {opt }}$ increases as the number of active users increases. This is consistent with Fig. 8.

Finally, Fig. 10 shows the comparison of the analytical and simulation BERs of the full 2-D-RAKE receiver for both cases of ideal CSI and imperfect channel estimation. It can be seen 
that the simulation results coincide approximately with the analytical results.

\section{CONCLUSION}

In this paper, the downlink BER performance of a CDMA system with TD-STBC is investigated under frequency-selective fading channels. A 2-D-RAKE receiver is exploited to collect both spatial and multipath diversity gain. Under the assumption that the ideal CSI is available at the receiver, some closed-form BER results are obtained when the pair of channels $h_{1, l}$ and $h_{2, l}$ are mutually independent. Without demand for additional spreading code sequence, a pilot-signal-assisted channel-estimation approach is exploited. It is illustrated that the imperfect channel estimation significantly degrades the performance of the TD-STBC system. Furthermore, the LPF can be employed to increase the accuracy of channel estimates, thus improving the BER performance under the slow-fading channel scenario. The following conclusions are drawn.

1) The RAKE receiver improves performance for both imperfect channel estimation and ideal CSI when the number of fingers increases. However, the improvement in BER for imperfect channel estimation is not as much as that for ideal CSI. Both equal gain combining and general selection combining improve performance significantly. However, MR-GSC outperforms MR-EGC.

2) When the number of taps of LPF $N_{P}$ increases from a small number, the performance of imperfect channel estimation improves significantly. However, increasing $N_{P}$ further only improves performance slightly. For example, increasing $N_{P}$ beyond 20 improves BER performance insignificantly.

3) The optimal power ratio of the pilot to one data channel increases as the number of active users increases. For $K=$ 10 , the optimal power ratio $g$ is around $3 \mathrm{~dB}$.

\section{APPENDIX \\ DERIVATION OF BER FOR AN MR-GSC RECEIVER WITH IDEAL CSI}

When $L_{c}=2$, the two strongest paths are selected and combined so that the closed-form BER can be manipulated as [20]

$$
\begin{aligned}
P_{e, \mathrm{GSC}}^{\langle 2\rangle}= & \left(\begin{array}{c}
L \\
2
\end{array}\right) \cdot 2 \cdot \int_{0}^{\infty} \int_{\gamma_{2: L}}^{\infty}\left[F_{\gamma_{l}}\left(\gamma_{2: L}\right)\right]^{L-2} \\
& \cdot p_{\gamma_{l}}\left(\gamma_{1: L}\right) \cdot p_{\gamma_{l}}\left(\gamma_{2: L}\right) \\
& \cdot Q\left(\sqrt{\left.2 \cdot \sum_{l=1}^{2} \gamma_{l: L}\right) \cdot d \gamma_{1: L} \cdot d \gamma_{2: L}}\right. \\
= & \frac{L(L-1)}{2} \cdot\left\{P_{e 1}^{\langle 2\rangle}+\sum_{m=1}^{L-2} \sum_{n=0}^{m}\left(\begin{array}{c}
L-2 \\
m
\end{array}\right)\right. \\
& \left.\cdot\left(\begin{array}{c}
m \\
n
\end{array}\right) \cdot(-1)^{m} \cdot\left[P_{e 2}^{\langle 2\rangle}+P_{e 3}^{\langle 2\rangle}\right]\right\}
\end{aligned}
$$

where, by the definition

$$
P_{e 1}^{\langle 2\rangle}=\frac{1}{2}-\frac{16 \bar{\gamma}_{c}^{3}+56 \bar{\gamma}_{c}^{2}+70 \bar{\gamma}_{c}+35}{32 \cdot\left(1+\bar{\gamma}_{c}\right)^{3} \cdot \sqrt{1+1 / \bar{\gamma}_{c}}}
$$

$$
\begin{aligned}
P_{e 2}^{\langle 2\rangle}= & -\frac{(2 m+2) \cdot \bar{\gamma}_{c}+m^{2}+4 m+2}{m \cdot(m+2) \cdot\left(1+\bar{\gamma}_{c}\right)} \\
& \cdot \sqrt{\frac{2}{\pi}} \cdot \frac{\Gamma(n+5 / 2) \cdot \bar{\gamma}_{c}^{1 / 2}}{\left(m+2+2 \bar{\gamma}_{c}\right)^{n+5 / 2}} \\
P_{e 3}^{\langle 2\rangle}= & \frac{2(n-m+2)\left(1+\bar{\gamma}_{c}\right)-m}{2 \cdot m^{n+3} \cdot\left(1+\bar{\gamma}_{c}\right) \cdot \sqrt{1+1 / \bar{\gamma}_{c}}} \\
& \cdot I_{A}\left(n+1, \frac{m}{2+2 \bar{\gamma}_{c}}\right)+\frac{n+m+4}{(m+2)^{n+3}} \\
& \cdot I_{A}\left(n+1, \frac{m+2}{2 \bar{\gamma}_{c}}\right)
\end{aligned}
$$

where $\bar{\gamma}_{c}$ is given by (30) and the function $I_{A}(\cdot, \cdot)$ is defined in (44).

As $L_{c}=3$, the three strongest paths are selected and combined so that the closed-form BER can be manipulated as

$$
\begin{aligned}
P_{e, \mathrm{GSC}}^{\langle 3\rangle}= & 3 ! \cdot\left(\begin{array}{c}
L \\
3
\end{array}\right) \cdot \int_{0}^{\infty} \int_{\gamma_{3: L}}^{\infty} \int_{\gamma_{2: L}}^{\infty}\left[F_{\gamma_{l}}\left(\gamma_{3: L}\right)\right]^{L-3} \\
& \cdot \prod_{l=1}^{3} p_{\gamma_{l}}\left(\gamma_{l: L}\right) \cdot Q\left(\sqrt{2 \cdot \sum_{l=1}^{3} \gamma_{l: L}}\right) \\
& \cdot d \gamma_{1: L} \cdot d \gamma_{2: L} \cdot d \gamma_{3: L} \\
= & \frac{3 !}{2} \cdot\left(\begin{array}{c}
L \\
3
\end{array}\right) \cdot\left\{P_{e 1}^{\langle 3\rangle}+\sum_{m=1}^{L-3} \sum_{n=0}^{m}\left(\begin{array}{c}
L-3 \\
m
\end{array}\right) \cdot\left(\begin{array}{c}
m \\
n
\end{array}\right)\right. \\
& \cdot(-1)^{m} \cdot P_{e 2}^{\langle 3\rangle}+\sum_{m=0}^{L-3} \sum_{n=0}^{m}\left(\begin{array}{c}
L-3 \\
m
\end{array}\right) \\
& \left.\cdot\left(\begin{array}{c}
m \\
n
\end{array}\right) \cdot(-1)^{m} \cdot\left[P_{e 3}^{\langle 3\rangle}+P_{e 4}^{\langle 3\rangle}\right]\right\}
\end{aligned}
$$

where, by the definition

$$
\begin{aligned}
P_{e 1}^{\langle 3\rangle}= & -\frac{288 \bar{\gamma}_{c}^{3}+848 \bar{\gamma}_{c}^{2}+790 \bar{\gamma}_{c}+477}{13824 \cdot\left(1+\bar{\gamma}_{c}\right)^{5} \cdot \sqrt{1+1 / \bar{\gamma}_{c}}} \\
P_{e 2}^{\langle 3\rangle}= & -\frac{3}{4 \cdot m^{n+5} \cdot \sqrt{1+1 / \bar{\gamma}_{c}}} \\
& \cdot I_{A}\left(n+4, \frac{m}{3+3 \bar{\gamma}_{c}}\right) \\
& +\frac{2 \bar{\gamma}_{c}+3}{8 \cdot m^{n+4} \cdot\left(1+\bar{\gamma}_{c}\right) \cdot \sqrt{1+1 / \bar{\gamma}_{c}}} \\
& \cdot I_{A}\left(n+3, \frac{m}{3+3 \bar{\gamma}_{c}}\right) \\
& +\frac{8 \bar{\gamma}_{c}^{2}+20 \bar{\gamma}_{c}+15}{16 \cdot m^{n+3} \cdot\left(1+\bar{\gamma}_{c}\right)^{2} \cdot \sqrt{1+1 / \bar{\gamma}_{c}}} \\
& \cdot I_{A}\left(n+2, \frac{m}{3+3 \bar{\gamma}_{c}}\right) \\
& -\frac{16 \bar{\gamma}_{c}^{3}+56 \bar{\gamma}_{c}^{2}+70 \bar{\gamma}_{c}+35}{32 \cdot m^{n+2} \cdot\left(1+\bar{\gamma}_{c}\right)^{3} \cdot \sqrt{1+1 / \bar{\gamma}_{c}}} \\
& \cdot I_{A}\left(n+1, \frac{m}{3+3 \bar{\gamma}_{c}}\right)
\end{aligned}
$$




$$
\begin{aligned}
P_{e 3}^{\langle 3\rangle}= & \frac{1}{2 \cdot(m+3)^{n+4}} \cdot I_{A}\left(n+3, \frac{m+3}{3 \bar{\gamma}_{c}}\right) \\
& +\frac{1}{(m+3)^{n+3}} \cdot I_{A}\left(n+2, \frac{m+3}{3 \bar{\gamma}_{c}}\right) \\
& +\frac{1}{2 \cdot(m+3)^{n+2}} \cdot I_{A}\left(n+1, \frac{m+3}{3 \bar{\gamma}_{c}}\right) \\
P_{e 4}^{\langle 3\rangle}= & \sqrt{\frac{3 \bar{\gamma}_{c}}{\pi} \cdot\left[\frac{1}{4 \cdot\left(1+\bar{\gamma}_{c}\right)} \cdot \frac{\Gamma(n+9 / 2)}{\left(m+3+3 \bar{\gamma}_{c}\right)^{n+9 / 2}}\right.} \\
& -\frac{2+\bar{\gamma}_{c}}{4 \cdot\left(1+\bar{\gamma}_{c}\right)^{2}} \cdot \frac{\left(m+3+3 \bar{\gamma}_{c}\right)^{n+7 / 2}}{(n+7 / 2)} \\
& \left.-\frac{19+22 \bar{\gamma}_{c}+8 \bar{\gamma}_{c}^{2}}{16 \cdot\left(1+\bar{\gamma}_{c}\right)^{3}} \cdot \frac{\Gamma(n+5 / 2)}{\left(m+3+3 \bar{\gamma}_{c}\right)^{n+5 / 2}}\right] .
\end{aligned}
$$

\section{REFERENCES}

[1] J. H. Winters, "The diversity gain of transmit diversity in wireless systems with Rayleigh fading," IEEE Trans. Veh. Technol., vol. 47, pp. 119-123, Feb. 1998.

[2] V. Tarokh, N. Seshadri, and A. R. Calderbank, "Space-time codes for high data rate wireless communication: Performance criterion and code construction," IEEE Trans. Inform. Theory, vol. 44, pp. 744-765, Mar. 1998.

[3] V. Tarokh, H. Jafarkhani, and A. R. Calderbank, "Space-time block codes from orthogonal designs," IEEE Trans. Inform. Theory, vol. 45, pp. 1456-1467, July 1999.

[4] S. M. Alamouti, "A simple transmit diversity technique for wireless communications," IEEE J. Select. Areas Commun., vol. 16, pp. 1451-1458, Oct. 1998

[5] B. Hochwald, T. L. Marzetta, and C. B. Papadias, "A transmit diversity scheme for wideband CDMA systems based on space-time spreading," IEEE J. Select. Areas Commun., vol. 19, pp. 48-60, Jan. 2001.

[6] Physical Channel and Mapping of Transport Channels Onto Physical Channels (FDD), Dec. 2002

[7] Physical Layer Standard for CDMA2000 Spread Spectrum Systems, Release C, Version 1.0, May 2002.

[8] V. Tarokh, S. M. Alamouti, and P. Poon, "New detection schemes for transmit diversity with no channel estimation," in Proc. IEEE Int. Conf. Universal Personal Commun., vol. 2, Oct. 1998, pp. 917-920.

[9] B. A. Bjerke, J. G. Proakis, and Z. Zvonar, "Antenna diversity combining schemes for WCDMA systems in fading multipath channels," in Proc. 52nd IEEE Vehicular Technology Conf., vol. 1, Sept. 2000, pp. 421-428.

[10] A. Vielmon, Y. Li, and J. R. Barry, "Performance of transmit diversity over time-varying Rayleigh-fading channels," in Proc. IEEE Global Telecommun. Conf., Nov. 2001, pp. 3242-3246.

[11] V. Tarokh, A. Naguib, N. Seshadri, and A. R. Calderbank, "Space-time codes for high data rate wireless communication: Performance criteria in the presence of channel estimation errors, mobility, and multiple paths," IEEE Trans. Commun., vol. 47, pp. 199-207, Feb. 1999.

[12] R. M. Buehrer and N. A. Kumar, "The impact of channel estimation error on space-time block codes," in Proc. 56th IEEE Vehicular Technology Conf., vol. 3, Sept. 2002, pp. 1921-1925.

[13] J. Wang and J. Chen, "Performance of wideband CDMA systems with complex spreading and imperfect channel estimation," IEEE J. Select. Areas Commun., vol. 19, pp. 152-163, Jan. 2001.
[14] J. C. Liberti Jr. and T. S. Rappaport, Smart Antennas for Wireless Communications: IS-95 and Third Generation CDMA Applications. Englewood Cliffs, NJ: Prentice-Hall, 1999.

[15] J. G. Proakis, "Digital communications through fading multipath channels," in Digital Communications, 4th ed. New York: McGraw-Hill, 2001, ch. 14, pp. 824-825.

[16] H. A. David, Order Statistics, 2nd ed. New York, NY: Wiley, 1981

[17] B. C. Arnold, N. Balakrishnan, and H. N. Nagaraja, A First Course in Order Statistics. New York: Wiley, 1992.

[18] M. K. Simon and M. S. Alouini, Digital Communication Over Fading Channels-A Unified Approach to Performance Analysis. New York: Wiley, 2000.

[19] X. Y. Wang, "Transmit Diversity in CDMA for wireless communications," Ph.D. dissertation, Univ. Hong Kong, Hong Kong, 2003.

[20] I. S. Gradshteyn and I. M. Ryzhik, Table of Integrals, Series, and Products, 6th ed. San Diego, CA: Academic, 2000.

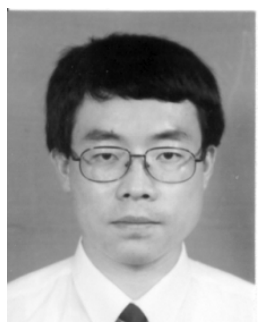

Xiangyang Wang received the B.S. degree in information engineering from Southwest Jiaotong University, Chengdu, China, in 1993, the M.S. degree in signal and information processing from Southeast University, Nanjing, China, in 1996, and the Ph.D. degree in electrical engineering from the University of Hong Kong, Hong Kong, in 2003.

From 1996 to 2000, he was with the National Mobile Communications Research Laboratory, Southeast University, as a Lecturer involved in the development and design of CDMA communication prototype systems. Since 2003, he has been with Southeast University as a Lecturer. His research interests include mobile communication systems, space-time signal processing, B3G/4G system, and the VLSI implementation of wireless systems.

Jiangzhou Wang (M'91-SM'94) received the B.S. and M.S. degrees from Xidian University, Xian, China, in 1983 and 1985, respectively, and the Ph.D. degree (with Greatest Distinction) from the University of Ghent, Ghent, Belgium, in 1990, all in electrical engineering.

From 1990 to 1992, he was a Postdoctoral Fellow with the University of California, San Diego, where he worked on the research and development of cellular CDMA systems. From 1992 to 1995, he was a Senior System Engineer with Rockwell International Corporation, Newport Beach, CA, where he worked on the development and system design of wireless communications. Since 1995, he has been with the University of Hong Kong, Hong Kong, where he currently is a Coordinator of Telecommunications Group and an Associate Professor. He was a Visiting Professor with NTT DoCoMo, Japan. He has published over 100 papers, including more than 20 IEEE transactions/journal papers in the areas of wireless mobile and spread-spectrum communications. He has written/edited two books, entitled Broadband Wireless Communications (Boston, MA: Kluwer, 2001) and Advances in $3 G$ Enhanced Technologies for Wireless Communications (Norwood, MA: Artech House, 2002). He holds one U.S. patent in the GSM system.

Dr. Wang is an Editor for the IEEE TRANSACTIONS ON COMMUNICATIONS and a Guest Editor for IEEE JouRnal on SELECTED AREAS IN COMMUNICATIONS. He was a Technical Chairman of the IEEE Workshop on 3G Mobile Communications in 2000 and is listed in Who's Who in the World. 